\title{
High-Fidelity 3D-Nanoprinting via Focused Electron Beams: Computer-Aided Design (3BID)
}

\author{
Jason D. Fowlkes, ${ }^{*},{ }^{\dagger} \ddagger \odot$ R. Winkler, ${ }^{\S}$ Brett B. Lewis, ${ }^{\ddagger}$ A. Fernández-Pacheco, ${ }^{\perp} \odot$ L. Skoric, ${ }^{\perp}$ \\ D. Sanz-Hernández, ${ }^{\perp \odot}$ Michael G. Stanford, ${ }^{\ddagger \odot}$ Eva Mutunga, ${ }^{\#}$ P. D. Rack, ${ }^{\dagger,}$ and H. Plank ${ }^{*},, \| \odot$ \\ ${ }^{\dagger}$ Center for Nanophase Materials Sciences, Oak Ridge National Laboratory, Oak Ridge, Tennessee 37831, United States \\ ${ }^{\ddagger}$ Materials Science and Engineering Department and ${ }^{\#}$ Bredesen Center for Interdisciplinary Research, The University of Tennessee, \\ Knoxville, Tennessee 37996, United States \\ ${ }^{\S}$ Graz Centre for Electron Microscopy, Steyrergasse 17, 8010 Graz, Austria \\ "Institute for Electron Microscopy and Nanoanalysis, Graz University of Technology, Steyrergasse 17, 8010 Graz, Austria \\ ${ }^{\perp}$ Cavendish Laboratory, University of Cambridge, JJ Thomson Avenue, Cambridge CB3 OHE, U.K.
}

\section{Supporting Information}

\begin{abstract}
Currently, there are few techniques that allow true 3D-printing on the nanoscale. The most promising candidate to fill this void is focused electron-beam-induced deposition (FEBID), a resist-free, nanofabrication compatible, direct-write method. The basic working principles of a computer-aided design (CAD) program (3BID) enabling 3D-FEBID is presented and simultaneously released for download. The 3BID capability significantly expands the currently limited toolbox for 3D-nanoprinting, providing access to geometries for optoelectronic, plasmonic, and

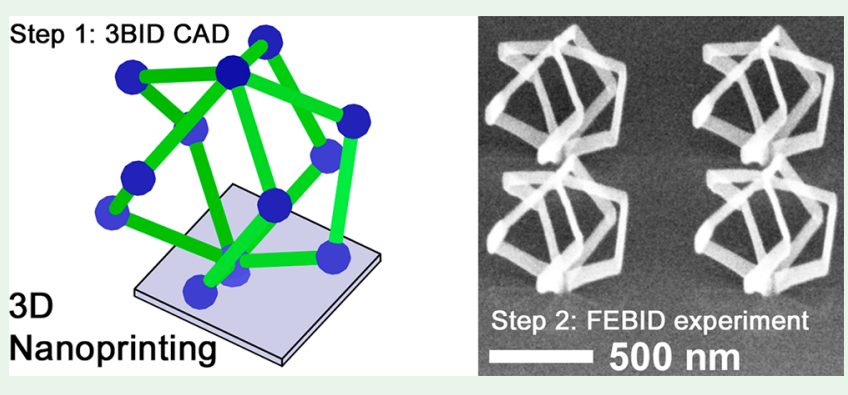
nanomagnetic applications that were previously unattainable due to the lack of a suitable method for synthesis. The CAD approach supplants trial and error toward more precise/accurate FEBID required for real applications/device prototyping.

KEYWORDS: 3D-nanoprinting, computer-aided design, direct write, additive manufacturing, focused electron beam induced deposition
\end{abstract}

\section{INTRODUCTION}

Nanotechnology urgently requires methods for direct 3Dfabrication, both to access emergent materials properties derived from $3 \mathrm{D}$-architectures ${ }^{1-3}$ and potentially as an alternative strategy to in-plane miniaturization. Direct-write, additive manufacturing using layer-by-layer deposition, or printing, has emerged in recent years as the method to deposit $3 \mathrm{D}$-objects from the macro to microscales. ${ }^{4}$ Unfortunately, 3Dnanoprinting methods are few. ${ }^{5,6}$ Focused electron-beaminduced deposition (FEBID) is one such 3D-nanoprinting technique where a nanoscale focused electron beam dissociates surface bound precursor molecules. ${ }^{7,8}$ FEBID is resist-free and direct-write additive; the precursor is typically an organometallic molecule intended to deposit metal or dielectric materials in response to electron driven dissociation.

After overcoming historical constraints such as deposit purity, FEBID has seen resurgence. These challenges are summarized succinctly in ref 9. In situ chemical methods have emerged to ensure material purity in the final deposit, since organometallics used in FEBID often yield carbon composites, when metal or metal oxides are intended, because precursors had been initially engineered for thermal CVD. ${ }^{10-14}$ Comprehensive surface science studies have recently provided new insights into precursor dissociation under electron irradiation. ${ }^{15-22}$ Precursors tailored for FEBID are anticipated soon.

High-purity FEBID opened the door for the translation to $3 \mathrm{D}$-nanoprinting. As early as 20 years ago, the potential for 3Ddeposition was already recognized. ${ }^{23-26}$ Complex 3D-nanostructures were also fabricated by focused ion-beam-induced deposition (FIBID), ${ }^{27-29}$ and a 3D-computer-aided design (CAD) environment for use with ions was even created. ${ }^{30}$ Over the ensuing years, 3D-FEBID was demonstrated by Bret/ Utke, $^{31-33}$ Molhave, $^{34}$ Gazzadi, $^{35}$ and Matsui $^{36}$ (FIBID), providing the first realizations of controlled growth. Key in all of these studies was recognition of the critical role of beam speed, in the context of a complex electron-solid interaction. More recently, beam scanning strategies tailored to minimize precursor surface depletion during FEBID $^{37-39}$ demonstrated increased FEBID efficiency and mitigated the evolution of complex nanoscale roughness on $2 \mathrm{D}$-surfaces. ${ }^{40}$ These accomplishments have led to an improved fundamental understanding of FEBID paving the way for stable 3D-growth.

Received: January 29, 2018

Accepted: February 14, 2018

Published: February 14, 2018 
Nanostructures created using 3D-FEBID include magnetic nanostructures $\left[\mathrm{Co}_{2}(\mathrm{CO})_{8}\right],{ }^{41,42}\left[\mathrm{HCo}_{3} \mathrm{Fe}(\mathrm{CO})_{12}\right],{ }^{43}$ chiral plasmonic nanohelices $\left[\mathrm{MeCpPt}^{\mathrm{IV}} \mathrm{Me}_{3}\right],{ }^{44}\left[\mathrm{Fe}_{2}(\mathrm{CO})_{9}\right]^{45} \mathrm{de}-$ rived vertical wires, core-shell nanohelices enabled using $\mathrm{SiO}_{x}$ scaffolds derived from $\left[\mathrm{Si}\left(\mathrm{OC}_{2} \mathrm{H}_{5}\right)_{4}\right],{ }^{46}$ gold nanohelices $\left[\mathrm{Me}_{2} \mathrm{Au}(\mathrm{acac})\right],{ }^{47}$ and $\mathrm{Co}_{3} \mathrm{Fe}$ nanocubes. ${ }^{48}$ Notably, a postdeposition purification process has been developed that transforms $\mathrm{AuC}_{x}$ composites into pure $\mathrm{Au} 3 \mathrm{D}$-architectures for plasmonic applications ${ }^{49}$ using $\mathrm{Me}_{2} \mathrm{Au}(\mathrm{acac})$. Concurrently, a simulation capable of predicting 3D-FEBID was demonstrated that could predict the 3D-FEBID of complex mesh/ lattice objects over hundreds of nanometers. ${ }^{50}$ Although powerful in its capabilities, this simulation requires a suitable $\mathrm{CAD}$ graphical user interface (GUI) to transform a scientific tool into a comprehensive package for the generic creation of complex 3D-nanoarchitectures using FEBID. Here, the details of the CAD environment (3BID) are presented together with practical applications and the release of the freeware package to provide the 3D-FEBID capability to the broader community in diverse fields in research and development. In addition, the impact of the beam exposure sequence is discussed, and tools are provided to compensate for exposure artifacts that would otherwise yield a 3D-geometry different from the intended 3DCAD model. FEBID simulations provide insight on the physical origins of exposure artifacts that are a consequence of exposure sequence. FEBID experiments target the fundamental 3Dbuilding block, a vertical pillar, supporting an overhanging nanowire, or so-called segment. Mastery over the deposition of this composite element, for a range of segment angles spanning from 0 to $90^{\circ}$, unlocks the ability to deposit complex 3D-mesh style objects. Finally, step-by-step methods are provided in the Supporting Information as PDFs and videos, comprehensively covering the use of the 3BID program.

\section{BACKGROUND}

\subsection{Focused Electron-Beam-Induced Deposition} (FEBID). FEBID consists of the localized dissociation of a precursor gas via electron beam irradiation. The precursor is delivered to the substrate by vapor phase impingement using a capillary nozzle inserted near the beam impact region (BIR). The precursor continuously adsorbs on the substrate as well as on newly formed deposited material under repetitive electron exposure, accumulating a microscopic solid deposit. A steady flux of vapor phase precursor is required to achieve continuous growth. The immersion of the growing deposit in the vapor phase provides a nearly constant flux of precursor on all deposit surfaces, regardless of their spatial orientation, enabling nearly three-dimensional (3D) isotropic deposition if beam/patterning parameters do not lead to significant precursor depletion.

In general, secondary electrons (SE) are the most energetically favorable electron species for precursor dissociation and are created during inelastic electron-solid collisions between primary/incident electrons and the deposit solid. SE energies span the energy range of up to $50 \mathrm{eV}$, while incident primary electrons in the initial beam have energies on the order of several to tens of $\mathrm{keV}$, depending on the primary electron beam settings.

High-energy primary electrons can have an elastic scattering range of micrometers in the solid which seemingly limits final deposit resolution to the micrometer scale-the so-called electron scattering proximity effect. However, the relatively low energy of SEs limits their scattering range (mean free path) in the solid to the nanometer scale. As a result, SEs are readily reabsorbed by the solid. Thus, even though SEs are liberated continuously over the cumulative primary electron path, which can span micrometers, only SEs generated in the near surface region can intersect the surface and induce dissociation/ deposition. Importantly, the SE current density produced at the location of primary beam impact far exceeds the stray SE current density at other surfaces on the deposit due to beam divergence caused by elastic electron scattering in the deposit. The secondary electrons generated at these remote locations are generated by backscattered and forward-scattered primary electrons called type-II secondary electrons. If the rate of precursor delivery approaches the dissociation rate in the beam impact region, FEBID resolution is nanoscale with a lateral resolution approaching the beam size. In this case, SE driven dissociation is localized mostly to the primary electron beam impact region - the highly localized, nanoscale concentration of secondary electron flux makes 3D-FEBID possible. A 3Dpatterning strategy will now be described for use with FEBID but is also applicable for use with ions (FIBID). For brevity, the program description will be limited to use with FEBID, because ion-induced sputtering can complicate design for FIBID patterning, which has not yet been included in the current program.

2.2. 3D-Nanofabrication with an Electron Beam. The nature of electron exposure using conventional electron optics simplifies 3D-deposition into a 2D-exposure patterning sequence projected into the optical focal plane. Growth in the vertical dimension, orthogonal to the focal plane, naturally ensues if the primary electron beam maintains intersection with the growing deposit. Typically, the primary charged particle beam impacts the substrate at normal incidence, along the $z$ axis. Previous studies have demonstrated a range of beam patterning velocities ${ }^{32,35}$ demonstrating suspended element growth.

The electron beam size at focus determines the primary electron beam current density. Consider FEBID during stationary electron beam exposure for a primary electron dwell time of $\left(\tau_{\mathrm{d}}\right)$. The SE current density is related to the primary value through the secondary electron yield at beam impact $\left(\delta_{I}\right)$ as $i_{\mathrm{SE}}=\delta_{I} \times i_{b}$. The SE current density controls the mean FEBID vertical growth rate as a direct multiplication factor

$$
\bar{v}=\frac{1}{\tau_{d}} \int_{0}^{\tau_{d}} i_{\mathrm{SE}}(t) \sigma \Omega_{d} C_{s}(t) \mathrm{d} t
$$

where $(\bar{v})$ is the average vertical growth rate in $[\mathrm{m} / \mathrm{s}],\left(i_{\mathrm{SE}}\right)$ is the SE current density $\left[\mathrm{e}^{-} /\left(\mathrm{m}^{2} \mathrm{~s}\right)\right]$ at beam impact, $(\sigma)$ is the electron impact dissociation cross section $\left[\mathrm{m}^{2} / \mathrm{e}^{-}\right],\left(\Omega_{d}\right)$ is the volume per deposit molecule $\left[\mathrm{m}^{3} /\right.$ molecule $]$, and $\left(C_{s}\right)$ is the surface concentration of bound precursor molecules [molecules $\left./ \mathrm{m}^{2}\right]$. The time dependence of $i_{\mathrm{SE}}(t)$ arises from the dynamic convolution of the $3 \mathrm{D}$ secondary-electron-emission profile with a deposit of changing shape. In turn, this induces a time-dependent depletion of precursor at the BIR leading to $C_{s}(t)$.

The relatively large depth of field produced in electron optical columns makes it possible to assume a constant lateral beam size in the vertical coordinate, at least within several micrometers ${ }^{50}$ above and below the focal plane. Beam refocus is therefore not required during $3 \mathrm{D}$-deposition within this range. The rate of vertical growth is controlled by eq 1 . FEBID can be extended into the lateral coordinate by implementing a primary electron beam patterning velocity in the focal plane. 
The 3D-FEBID of mesh/lattice nanostructures is possible due to a reliable correlation between primary beam velocity and the angle $(\zeta)$ that a linear mesh element makes with respect to its projection in the focal plane as schematically shown in Figure $1 \mathrm{a} . \zeta$ is defined as the "segment angle". The primary

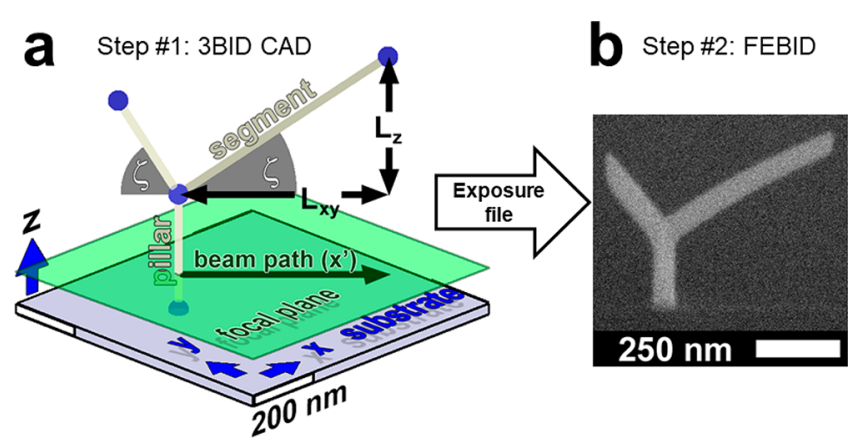

Figure 1. (a) Computer-aided design $(\mathrm{CAD})$ of a pillar $\left(\zeta=90^{\circ}\right)$ element supporting two segment $\left(\zeta<90^{\circ}\right)$ type elements. These elements represent the basic building blocks required to deposit complex, mesh style objects using focused electron-beam-induced deposition (FEBID). A CAD program called "3BID", an acronym for 3D-FEBID, provides a graphical user interface (GUI)-based program to create 3D-FEBID exposure files for experiments. (b) FEBID of the exposure, or stream, file based on the design shown in (a). The stream file contains a list of beam positions $\left(x_{b}, y_{b}\right)$ and dwell times $\left(\tau_{d}\right)$ for each primary electron beam exposure. The 3D-object was exposed using an acceleration voltage of $30 \mathrm{keV}$, a beam current of $31 \mathrm{pA}$, and the precursor $\mathrm{MeCp}\left(\mathrm{Pt}^{\mathrm{IV}}\right) \mathrm{Me}_{3}$. The step-by-step $\mathrm{CAD}$ of the branching nanostructure can be viewed in Method \#7 (video): NanoBranchBasic CAD.

electron beam patterning velocity $\left(v_{b}\right)$ in the focal plane $(x, y)$ is defined as

$$
v_{b}=\frac{\Lambda}{\tau_{d}}
$$

where $(\Lambda)$ is the pixel point pitch between exposure nodes. A single beam exposure indicates a stationary dwell for $\left(\tau_{d}\right)$ (eq 1).

In the computer-aided design (CAD) program described below, $(x, y, z)$ coordinates are first specified to define a deposit design. Elements are then defined that serve as linear interconnections spanning two specified coordinates yielding vertices. Vertical elements (with respect to the focal plane) are called pillars, while off-axis elements are termed segments. Segments are deposited during FEBID by scanning the electron beam along the $2 \mathrm{D}(x, y)$-projection of the segment into the focal plane. As will be shown, a central feature of the program is beam patterning velocity selection to ensure that the deposited element traces the intended 3D-path to the final coordinate position specified in the CAD. The impact of convergence semiangle on 3D-FEBID is explored in the companion article. ${ }^{51}$ The advantages of a relatively smaller probe size, achieved by increasing the convergence semiangle relative to standard operating conditions, includes a smaller segment cross-sectional area and a reduction in the codeposit volume produced on the underlying substrate due to a broadening of the transmitted beam, to name a few. ${ }^{51}$ However, these advantages are opposed by a reduced $z$-range of predictable 3D-FEBID above the substrate surface, a range over which the beam size can be approximated as constant. ${ }^{51}$ In addition, the influence of the magnetic immersion lens used for high-resolution deposition must be considered during the 3D-FEBID of magnetic materials.

Importantly, $\left(v_{b}\right)$ is set in the program by specification of $\left(\tau_{d}\right)$, for a fixed $(\Lambda) .{ }^{51}$ Ultimately, an experimentally derived calibration curve, or $(\zeta)$ versus $\left(\tau_{d}\right)$ plot, serves the critical role of determining the dwell time required for predictable $3 \mathrm{D}$ segment fabrication. Previous experiments have shown that a linear segment is produced at constant patterning velocity in the diffusion enhanced regime (DER) of FEBID $^{50}$ for segment lengths of several hundreds of nanometers. ${ }^{51}$ However, continued exposure leads to sublinear bending/bowing of the segment ( $\zeta$ becomes lower than the intended value). The $\mathrm{CAD}$ program anticipates the FEBID of linear elements, and as described below, the sublinear deformation may be treated with a segment compensation tool effectively accounting for segment deflection.

In summary, a 3D-object consisting of a mesh of interconnected wires is deposited by scanning the electron beam continuously through the projection of the object design in the imaging focal plane. By carefully choosing both the electron beam dwell time per segment and the order of segment exposure, a 3D-deposit is engineered to emerge from the substrate surface replicating the intended design (Figure 1b).

\section{METHODS: FEBID EXPERIMENTS}

A detailed accounting of the 3D-FEBID experimental procedure is provided in the companion paper. ${ }^{51}$ Similar conditions were used here. Specifically, a Thermo Fisher Scientific/FEI Nova 600 dual electron/ ion beam microscope was used in the experiments reported in this paper. Further, a primary electron energy of $30 \mathrm{keV}$ with a beam current of $31 \mathrm{pA}$ was used during deposition, where the supporting substrate was a $\mathrm{Si}$ wafer with a surface $\mathrm{SiO}_{2}$ layer nominally $5 \mathrm{~nm}$ thick. The Pt-based precursor $\mathrm{MeCpPt}^{\mathrm{IV}} \mathrm{Me}_{3}$ was used for deposition. The precursor reservoir temperature was set to $45{ }^{\circ} \mathrm{C}$. The gas injection system position is reported in more detail below, as two distinct GIS positions were used for deposition. Nonetheless, the GIS angle with respect to the substrate surface normal was $38^{\circ}$ for both cases.

\section{METHODS: FEBID COMPUTER-AIDED DESIGN}

The methods section contains references to video tutorials which provide step-by-step demonstrations of program use. The videos are silent. However, each video is accompanied by a written tutorial, which provides detailed explanations of each action performed in the video as well as explanations of $\mathrm{CAD}$ function.

Table 1 provides a summary of the variables pertaining to the methods.

The 3D-FEBID CAD (3BID) program was tested on a desktop PC (i7-6700 CPU, 3.4 GHz, 16GB RAM) using two versions of Matlab: the 32-bit version R2013b and 64-bit version R2017a. Performance was mostly instantaneous for objects with $\sim 200$ vertices or less. For these reasons, the program is not currently optimized for performance-redundant calculations are still present, inefficient for loop structures that have not been replaced with vectorization, logical indexing has not been fully implemented, and refactoring is not optimized. These changes will be made in later versions or upon request. The program will be available, uncompiled, in the public repository www.github.com (@FEBiD3D). The fitting procedure can be slow if a large number of parameters are selected. Runtime per parameter is $\sim 0.010 \mathrm{~s}$ per parameter. Also, it is recommended that $2 \mathrm{D}$ array sizes not exceed $1003 \mathrm{D}$-objects per exposure file.

4.1. 3D-Object Elements: Pillars and Segments. 3BID is designed to work best for mesh object deposition where a complete geometric description is provided by a list of vertices and interconnecting elements. The order of FEBID segment exposure requires careful consideration, beyond a simple list of vertices and 
Table 1. Variables Related to Mesh Geometry, Beam Scanning, and Beam Exposure ${ }^{a}$

\section{Primary electron beam scanning}

$\left(x_{b}, y_{b}\right)=$ beam position in the focal plane

$z_{b}=$ beam focal position relative to the substrate surface

$\Lambda=$ pixel point pitch, $\{\mathrm{PoP}\}$

$\tau_{d, i j}=$ beam dwell time per pixel (DT in companion paper)

$v_{b}=$ beam patterning velocity (PV in companion paper)

$\tau_{d, \text { max }}=$ maximum dwell time $\left(\tau_{d}>\tau_{d, \max }\right.$; achieved via multiple exposures per pixel)

Computer-aided design

$\left(x_{u}, y_{u}, z_{u}\right)=$ vertex coordinates in CAD, $\{x(\mathrm{~nm})\},\{y(\mathrm{~nm})\},\{z(\mathrm{~nm})\}$ $u=$ vertex index, $\{\mathrm{VOI}\}$

$\zeta_{i j}=$ segment angle with respect to the focal plane, $\{$ press + then $z(\mathrm{~nm})\}$

$i=$ exposure level index, $\{$ level $\}$

$j=$ segment index, per exposure level, $\{!$, blue columns $\}$

$\left(\Delta x_{i j}, \Delta y_{i j}, L_{z, i j}\right)=$ total linear displacements per linear segment $(i j)$

$\Delta x_{i j}=x_{u_{f}}-x_{u_{i}} ; \Delta y_{i j}=y_{u_{f}}-y_{u_{i}} ; \Delta z_{i j}=z_{u_{f}}-z_{u_{i}}$

$u_{i}=$ vertex with relatively smaller $(z)$ value, per segment, $(i)$

$u_{f}=$ vertex with relatively larger $(z)$ value, per segment, $(j)$

$L_{z, i j}=$ vertical component of segment length

$L_{x y, i j}=$ segment length projected into the focal plane

Exposure order notation

$P_{i j}=$ number of exposure pixels per segment

$Q_{i j}\left(\tau_{d, i j}\right)=$ number of beam exposures per pixel

$V_{i j}=$ number of beam exposures per segment

$M=$ number of exposure levels

$N_{i}=$ number of segments per exposure level

Calibration curve fitting

$\left(x^{\prime}, z\right)=$ coordinate system for fitting. A vertical plane $(z)$ oriented along the segment axis $\left(x^{\prime}\right)$

$v_{n}=$ maximum vertical growth rate, $\{\operatorname{VGR}(\mathrm{nm} / \mathrm{s})\}$

$r_{N}=$ initial nuclei radius at $\tau_{d}=0,\{\operatorname{adv} \rightarrow$ nuclei radius (fit) $\}$

$\Delta s=$ surface node spacing in $\left(x^{\prime}, z\right)$ plane

$K_{1}=$ fractional reduction in precursor coverage at $\tau_{d}=\infty,\{\mathrm{rPD}\}$

$K_{2}=$ precursor depletion rate, $\{\mathrm{pPD}\}$

${ }^{a}\{\text { Variable name as it appears on the GUI }\}^{b}\{$ bold face $\}$ variable name in companion paper

connections, due to details related to the electron-solid and precursor-solid interactions that occur by primary electron scattering during FEBID. ${ }^{33,52}$ First, the basic elements required to create a mesh object for FEBID are described below and presented in Figure 1a.

A linear element spanning two vertices is classified as either a segment or a pillar according to

$$
\text { segment: } 0 \leq \zeta<90^{\circ}
$$

$$
\text { pillar: } \zeta=90^{\circ}
$$

where the segment angle is defined mathematically by the displacements $\left(\Delta x, \Delta y, L_{z}\right)$ between vertices as

$$
\tan \zeta=\frac{L_{z}}{L_{x y}}
$$

and $L_{x y}=\left(\Delta x^{2}+\Delta y^{2}\right)^{1 / 2}$. For example, a horizontal segment with an orientation parallel to the substrate surface would have $\zeta=0^{\circ}$. See $[\mathrm{S} 1$ : Pillar vs segment] for a discussion of the selection of pillar versus segment exposure in the limit of the slope of the calibration curve tends to zero as $\zeta \rightarrow 90^{\circ}$, for the case of constant $(\Lambda)$. The element classification determines the mechanism of dwell time selection during exposure file creation in the program.

The two FEBID exposure element classifications are now discussed separately because the methods used to calculate the exposure dwell times for the two cases are markedly different.
4.2. FEBID Pillar: Calibration. Pillar exposure is conducted with a stationary primary electron beam fixed at $\left(x_{b}, y_{b}\right)$ in the focal plane. The characteristic dimension of the pillar is the total length $\left(L_{z}\right)$. Pillar exposure requires the user to determine the average vertical growth rate $\left(v_{n}\right)$ of a pillar from experiments, which is information that must be submitted into the program. The reason for the subscript $(n)$ indicates that the growth velocity is referenced along the wire axis of the pillar and segment type elements. For a pillar element, the growth direction is aligned along the $\mathrm{z}$-axis, normal to the substrate surface. A text edit input box is available on the GUI "VGR (nm/s)" for directly submitting $\left(v_{n}\right)$ into the program. During exposure file creation, the length of each individual pillar specified in the design is calculated. The total exposure dwell time required to deposit the pillar is then calculated $\left(L_{z} / v_{n}\right)$.

4.3. FEBID Pillar: CAD to Exposure. The total exposure dwell time for pillar (and segment) growth can exceed the maximum value allowed by instrumentation $\left(\tau_{d, \max }\right)$. This limitation is overcome by fracturing the exposure into a series of smaller, stationary exposures. The total number of exposures per pixel $\left(Q_{i j}\right)$ for the pillar type of element is calculated using [S2: Final exposure dwell time]

$$
Q_{i j}\left(\tau_{d, i j}\right)=\operatorname{ceil}\left|\frac{\tau_{d, i j}}{\tau_{d, \max }}\right|=\operatorname{ceil}\left|\frac{\frac{L_{z, i j}}{v_{n}}}{\tau_{d, \max }}\right|
$$

where the subscript $(i)$ refers the exposure level index, and $(j)$ is the pillar/segment number index (these indices are described in more detail later). The updated dwell time for the exposure file becomes

$$
\left.\tau_{\mathrm{d}, i j}\right|_{\operatorname{exp~file}}=\frac{\tau_{d, i j}}{Q_{i j}\left(\tau_{d, i j}\right)}
$$

4.4. FEBID Segments: Calibration. The characteristic parameter of a segment is the angle $(\zeta)$. An array of pillar supported segments covering the full range of segment angles from 0 to $\sim 90^{\circ}$ is deposited in order to derive a calibration curve. ${ }^{51}$ The calibration curve then makes it possible to convert the CAD into an FEBID exposure file.

During the calibration phase, each segment angle is measured and plotted versus the dwell time used to deposit the segment. The calibration data is then uploaded to the program. Later, when a complete 3D-design has been created, i.e., vertices defined and segment connections made, the program calculates $(\zeta)$ for each segment and then determines the required $\left(\tau_{d}\right)$ for the exposure file from the calibration curve.

This method creates a conundrum for first time users-no calibration exists for the creation of the segment array for calibration. Nonetheless, the beam patterning velocity range for high-resolution segment FEBID $(\Lambda=0.2-2 \mathrm{~nm})$ is narrow, spanning in the dwell time range of $1-100 \mathrm{~ms}$ as demonstrated on multiple microscopes (Thermo Fisher Scientific Nova and Helios, Zeiss NanoFab) with a localized gas-injection system, or GIS, capillary. ${ }^{51}$ The suggested electron beam and GIS settings are presented in Table 2 for several precursors. Thus, calibration curves included in the program are sufficient to deposit a segment array spanning at least a significant portion of the calibration range. The calibration curve shape ( $\zeta$ vs $\left.\tau_{d}\right)$ is seemingly universal (at least for microscopes and precursors tested here), which makes it possible to determine which portion of the curve is absent in a first trial calibration curve. Alternatively, using a global dwell time scaling factor in the program [S3: dwell time scaling], exposure files may be quickly re-exported to deposit a segment array spanning the desired range. Thus, the user should anticipate at least two trials of segment calibration exposure before their instrument is calibrated.

Regarding the specifics of the calibration array, in order to avoid possible effects from the substrate, the user is advised (although it is not required) to grow each segment suspended from a pillar. It is also suggested that each pillar should have a height $\left(L_{z}\right)$ equal to the anticipated mean mesh spacing in future 3D-designs (Figure S1). The pillar constitutes exposure level \#1. Exposure "levels" will be discussed in detail in a later section and refers to multiple numbers of segments 
Table 2. Instrumentation Settings That Impact 3D-FEBID, Potentially Requiring a New Calibration Curve ${ }^{a}$

Primary electron beam voltage

$E_{o}\left(10^{2} \mathrm{keV}\right)$

Primary electron beam current

$i_{b}\left(10^{2} \mathrm{pA}\right)$

Primary electron beam size

fwhm $\left(10^{\circ} \mathrm{nm}\right)$ : Full-width at half-maximum

Gas injection source (GIS) position

$\Delta x_{\mathrm{GIS}}\left(10^{1} \mu \mathrm{m}\right)$ : The $x$-component of the line extending from the beam impact region to the tip of the GIS. The tip of the GIS refers to the intersection of the GIS projection in an SEM image and the delivery axis of the nozzle.

$\Delta y_{\mathrm{GIS}}\left(10^{1} \mu \mathrm{m}\right)$ : The $y$-component of the line extending from the beam impact region to the tip of the GIS. The tip of the GIS refers to the intersection of the GIS projection in an SEM image and the delivery axis of the nozzle.

$\Delta z_{\mathrm{GIS}}\left(10^{2} \mu \mathrm{m}\right)$ : The distance measured from the bottom of the GIS nozzle to the substrate surface.

GIS angle $\left(38^{\circ}\right)$ : The angle of the GIS axis with respect to the vertical electron column

$T_{p}\left(45^{\circ} \mathrm{C}\right)$ : The precursor reservoir temperature, e.g., $\mathrm{Me}\left(\mathrm{Pt}^{\mathrm{IV}}\right) \mathrm{CpMe}_{3}$

OD $(860 \mu \mathrm{m})$ : The outer diameter of the precursor capillary nozzle

ID $(680 \mu \mathrm{m})$ : The inner diameter of the precursor capillary nozzle

Focal working distance (WD)

The focal WD $(5-10 \mathrm{~mm})$ controls both the minimum primary electron probe size as well as the convergence angle of the beam.

${ }^{a_{T}}$ The values described above should be recorded for each calibration curve.

and pillars that are exposed using alternating beam shots. A segment of a specified angle is produced on exposure level $\# 2$ by variation of $\left(v_{b}\right)$-an exposure sequence where the pixel point pitch is fixed $(\Lambda<2$ $\mathrm{nm}$ recommended $\left.{ }^{51}\right)$ and the beam dwell time is fixed for a given angle (eq 2). As demonstrated in the companion paper, for $\Lambda>2 \mathrm{~nm}$, relatively small variations in $(\Lambda)$ can significantly impact the segment angle. ${ }^{51}$ This general rule applies over a wide range of pattern velocities spanning from $50-125 \mathrm{~nm} / \mathrm{s}^{51}$ This is particularly important considering that most scanning electron microscopes and dual ion/electron instruments require manual focusing. Thus, at least some day-to-day variation in FEBID results should be anticipated. By selectively working in the $\Lambda<2 \mathrm{~nm}$ regime, one can avoid an additional source of experimental variation due to unstable patterning conditions. Again, Table 2 lists the recommended setting for each critical parameter that influences the segment angle. A sensitivity to instrumentation settings is a hallmark characteristic of 3D-FEBID. Instrumentation parameters that strongly influence the calibration curve are reviewed in [S5: Key parameters influencing 3D-FEBID].

4.5. FEBID Segments: CAD to Exposure. The pixel point pitch $(\Lambda)$ is a user-specified constant for each 3D-mesh object design. Upon segment definition by the user, the program calculates the length of the segment projected into the focal plane $\left(L_{x y}\right)$. The number of exposure pixels per segment $\left(P_{i j}\right)$ is then calculated according to

$$
P_{i j}=\frac{L_{x y}}{\Lambda}
$$

The total number of exposures per pixel $\left(Q_{i j}\right)$ for the segment $(j)$ type of element is calculated using [S6: Current $\tau_{d, \max }$ setting]

$$
Q_{i j}\left(\tau_{d, i j}\right)=\operatorname{ceil}\left|\frac{\tau_{d, i j}}{\tau_{d, \max }}\right|
$$

The dwell time is specific to each individual segment in the design $\left(\tau_{d, i j}\right)$ due to the unique segment angle that is specified for each segment connection. Therefore, the total number of exposures $\left(\mathrm{V}_{i j}\right)$ required to deposit the segment $(j)$ lying in the exposure level $(i)$ is

$$
V_{i j}=P_{i j} Q_{i j}
$$

In summary, the segment angle and $\left(L_{x y}\right)$ are calculated from the design prior to exposure file creation. $\zeta$ is then used to estimate the dwell time $\left(\tau_{d, i j}\right)$ from the calibration curve $\left(\zeta\right.$ vs $\left.\tau_{d}\right)$, either by interpolation of the experimental data [S7: Calibration data interpolation] or via a FEBID related data fit (later section). The segment is deposited using a total number of $\left(V_{i j}\right)$ exposures with a dwell time per exposure of $\left(\tau_{d, i j}\right) / Q_{i j}$.

The reader is encouraged to view the video and written tutorials covering basic CAD use (Method \#1 (video and tutorial)) and calibration file preparation (Method \#2 (video and tutorial)) at this point. In addition, Figure S2 provides an image of the graphical user interface (GUI) which cross references each GUI element to a specific method video.

4.6. 3D-Object Exposure Order: Continuous and Intermittent Exposure. Exposure order is a critical feature during 3D-FEBID, which acts to preserve beam-deposit intersection as deposition progresses normal to the focal plane. The user controls exposure order though the definition of exposure "levels". A level consists of one or more elements. Continuous, or serial, element exposure occurs for the case of one defined element per exposure level (Figure 2). In the case of multiple segments per exposure level, exposure proceeds in an intermittent, or interlacing, sequence. During intermittent exposure, beam exposure alternates between each element in the level as opposed to the continuous, uninterrupted exposure of each element (Figure 3). This can be expressed mathematically as
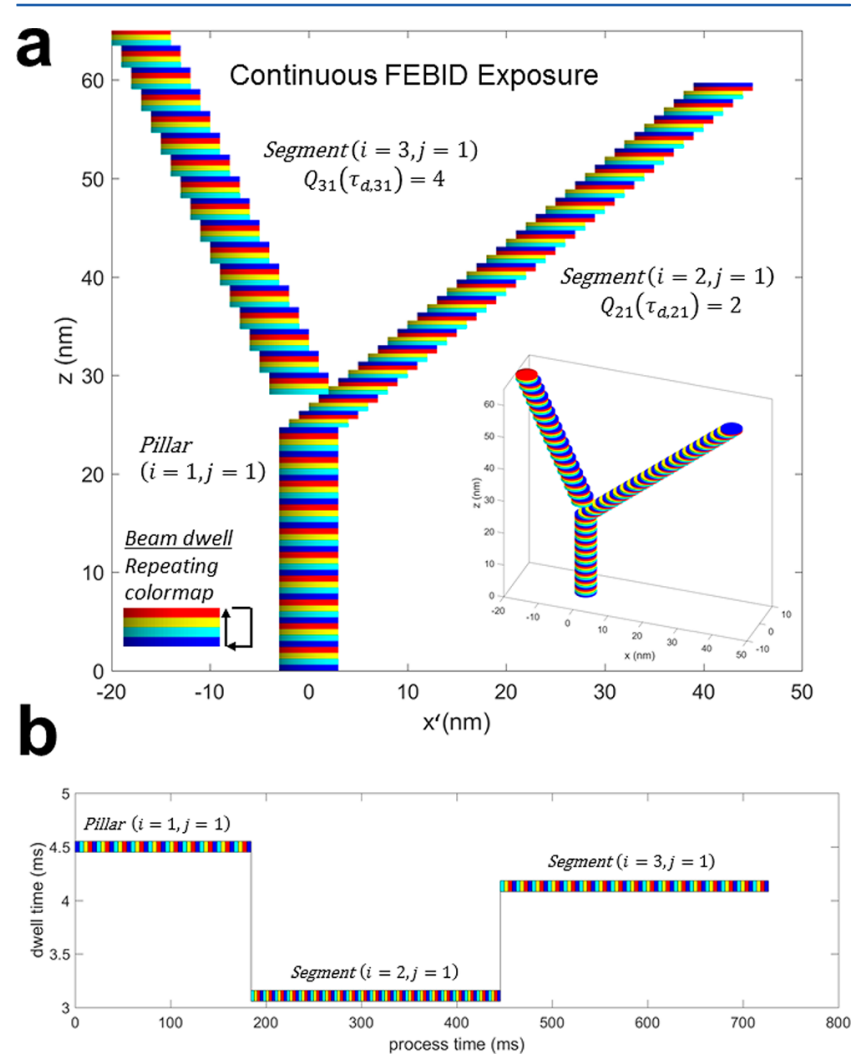

Figure 2. (a) Continuous (equivalent to serial) FEBID exposure scheme. A thin disc represents a single beam exposure. Disc thickness equals $\left(v_{n} * \tau_{d}\right)$, and the disc radius was set arbitrarily to $4 \mathrm{~nm}$ (on the order of the beam size). Overlapping exposure discs stack as a function of process time, providing a qualitative insight into the consequence of exposure sequencing during FEBID. The use of a repeating colormap reveals exposure sequencing (inset)-intermittent exposure (next figure) sequencing is evident when the stacking color sequence deviates from the colormap order. Continuous exposure is used for the entire structure in (a). (b) Exposure dwell time plotted as a function of the total exposure process time. The color of each exposure correlates with the disc stacking order in (a). The width of each colored exposure rectangle equals the dwell time. Continuous segment exposure is seen as long periods of process time with a common dwell time. 


$$
\sum_{i=1}^{M}\left[\sum_{j=1}^{N_{i}} \tau_{d, i j}\right]
$$
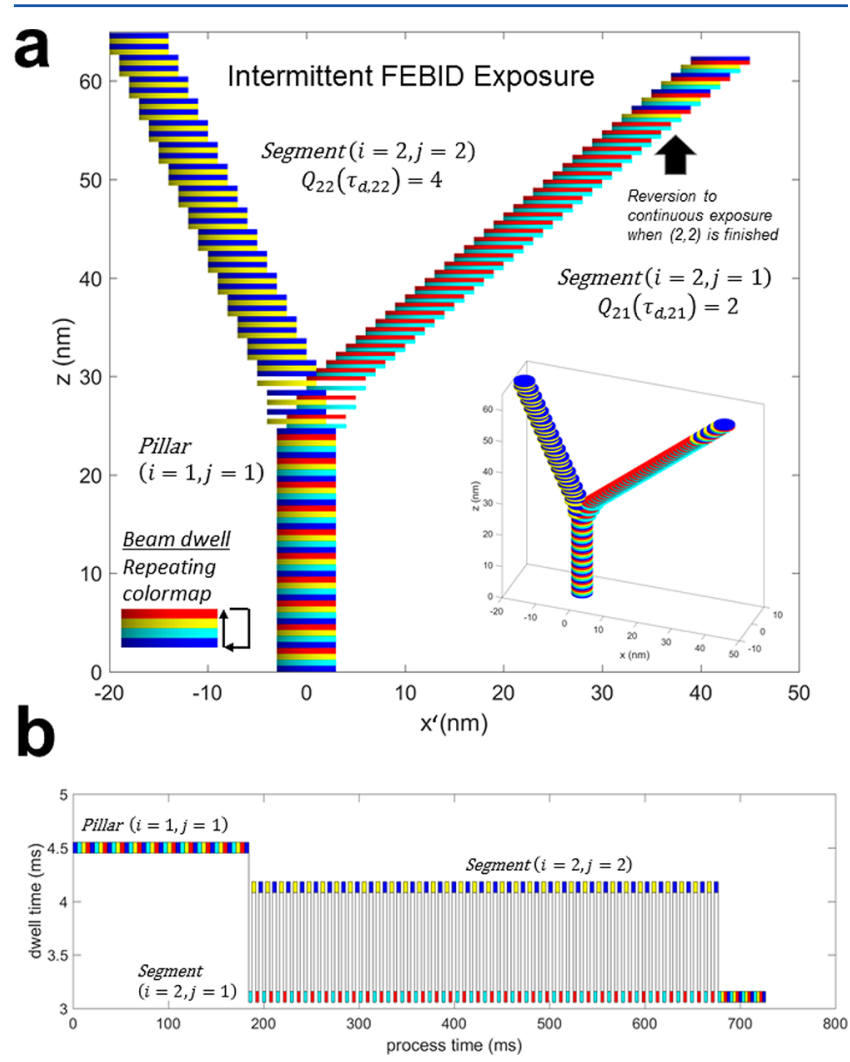

Figure 3. (a) Intermittent (equivalent to an interlacing) FEBID exposure scheme. A thin disc represents a single beam exposure. Disc thickness equals $\left(v_{n} * \tau_{d}\right)$, and the radius was set arbitrarily to $4 \mathrm{~nm}$ (on the order of the beam size). The alternating color scheme sequence in the colormap changes during intermittent exposure as exposure pixels cycle between $(i=2, j=1)$ and $(i=2, j=2)$. (b) Exposure dwell time plotted as a function of the total exposure process time. The color of each exposure correlates with the disc stacking shown in (a). Intermittent exposure is seen as oscillations in the exposure dwell time map between 180 and $670 \mathrm{~ms}$.

where $(M)$ is the total number of levels, and $(i)$ is the level index. The number of total segments per level is $\left(N_{i}\right)$. The subscript $(i)$ indicates that the number of segments can vary for each exposure level. Lastly, $(j)$ is the current segment index. Eq 10 can be further expanded to include the number of exposure pixels per pillar/segment and the influence of the maximum dwell time threshold described previouslythis is the master equation that governs exposure sequencing in the program

$$
\text { fabrication time }=\sum_{i=1}^{M}\left[\sum_{k=1}^{P_{i j}}\left\{\sum_{l=1}^{Q_{i j}\left(\tau_{d, i j}\right)}\left(\sum_{j=1}^{N_{i}}\left[\frac{\tau_{d, i j}\left(x_{b, i j k}, y_{b, i j k}\right)}{Q_{i j}\left(\tau_{d, i j}\right)}\right]\right)\right\}\right]
$$

With $(k)$, the variable cycles through each exposure pixel per segment, and $(l)$ cycles through the number of exposures required per pixel. The current exposure shot number per level per segment $(i j)$ is given by

$$
v_{i j k l}=(k-1) Q_{i j}\left(\tau_{d, i j}\right)+l
$$

In terms of exposure order, eqs 10 and 11 may be summarized in algorithm form as

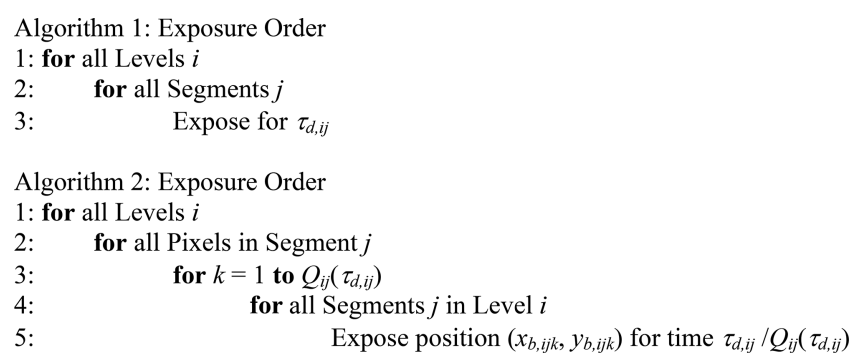

The intermittent exposure sequence is beneficial with regards to FEBID efficiency, as it stabilizes or shifts the working regime toward the electron-limited regime. Prolonged local electron dwell times, characteristic of serial exposure sequences in general, can induce timedependent precursor depletion. The most rapidly alternating element in eq 11 is the segment index, which exemplifies the importance placed on maximizing the precursor refresh time in the BIR. Surface coverage transients increase the probability of sublinear segment growth, which is undesirable when linear connectors between vertices are specified. The rapidly alternating intermittent exposure mode naturally introduces a precursor refresh time between adjacent exposure pixels per segment. Precursor refresh promotes the ELR condition ${ }^{39,52}$ when the precursor replenishment flux exceeds the dissociation flux either by surface diffusion and/or direct adsorption in the BIR. The simulation results presented here show localized depletion, confined to the BIR, for the instrumentation conditions reported here using the precursor $\mathrm{MeCp}\left(\mathrm{Pt}^{\mathrm{IV}}\right) \mathrm{Me}_{3}$. The simulation features have been described previously in ref 50 . Furthermore, the 3D-intermittent, or interlacing, strategy minimizes segment bending and drift issues. ${ }^{49}$ The reader should take note that the intermittent/interlacing approach has technical limitations due to a limiting speed for beam translation from pixel-to-pixel; long intermittent translations coupled with relatively brief dwell times can prevent stable 3D-nanofabrication.

4.7. 3D-FEBID Exposure Stream File. The CAD environment translates the design into a so-called stream file, which has been tested on a host of gas equipped dual electron/ion microscopes including the Thermo Fischer series of Nova and Helios microscopes as well as the Zeiss Orion NanoFab equipped with a NVPE patterning engine. The form of the stream file depends on the type of microscope. Nonetheless, a general exposure file is also created, regardless of the microscope type, which is a list of coordinates in sequential exposure order with the following format

$$
\left[\left(x_{1}, y_{1}, \tau_{d, 1}\right) ;\left(x_{2}, y_{2}, \tau_{d, 2}\right) ; \ldots\left(x_{n}, y_{n}, \tau_{d, n}\right)\right]
$$

and is exported as Exposure_File_Name_GEF.txt from the program if the generic name is chosen in the GUI.

\section{METHODS: CALIBRATION CURVE FITTING}

5.1. Simple FEBID Model. A nearly linear segment is produced by FEBID (over several hundreds of nanometers) under conditions of a constant beam patterning velocity in the limit of a beam displacement less than the primary electron beam size and a sufficiently high precursor flux to stabilize the working regime. A simple model was found to predict the segment angle over a wide range of beam patterning velocities. The purpose of the model is to fit sparsely populated experimental calibration curve data rather than to provide a simulation of expected results. For example, the model fails to account for the thickness and width of the segment. Conversely, the simulation reported in ref 50 provides a more accurate emulation of 3D-FEBID.

A simple "dwell and step" model was formulated to represent FEBID where a $2 \mathrm{D}$-surface is evolved as a function of stationary beam dwell time (Figure 4). Surface evolution is restricted to the lateral $\left(x^{\prime}\right)$ and vertical $(z)$ dimensions, because segment growth can be reduced to a planar problem (in this coordinate system, $\left(x^{\prime}\right)$ evolves along $L_{x y}$ ) [S9: The $\left(\mathrm{x}^{\prime}, \mathrm{z}\right)$ coordinate system]. At the conclusion of each primary beam dwell increment, the segment angle is taken as the angle between a vector lying in the focal plane and a line extending from the origin of the deposit to the surface position on the deposit at $x^{\prime}=\Lambda$. 

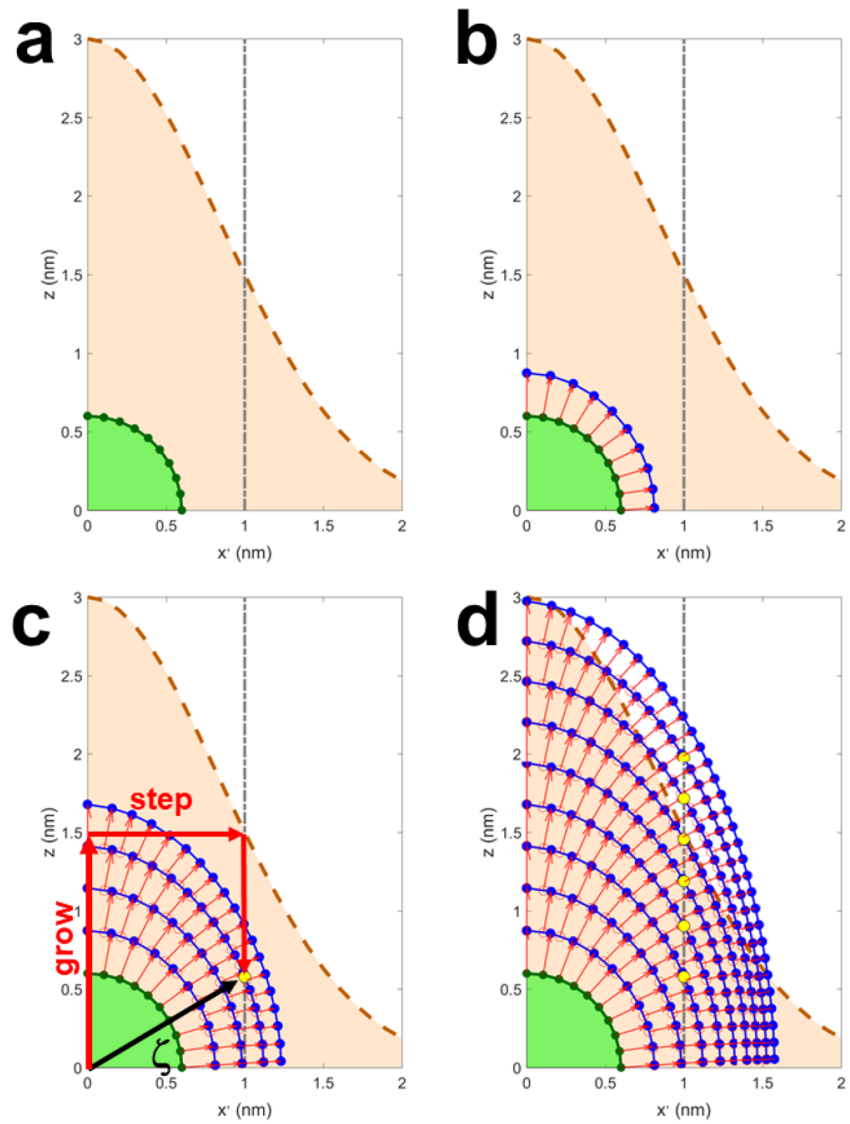

Figure 4. (a) Simple FEBID model used for fitting the calibration curve data starts with the definition of a pre-existing semicircular nuclei (green). This parameter captures variability in segment takeoff. The normalized Gaussian beam profile (orange) drives surface evolution, along with an exponential decay that emulates the possibility of precursor coverage depletion, which is not shown for clarity. The pixel point pitch for segment growth is shown as the hatched gray line. (b) Surface points (blue) defining the nuclei have evolved in a discrete time step $(\Delta t)$. Notice that the growth velocity depends on the $(x)$ position due to the beam profile, reflected by higher deposit volumes in the vertical direction compared with the $x^{\prime}$ direction. (c) The surface is rediscretized at each time step to preserve a nearly constant surface point spacing for numerical stability (open red data points). The yellow point is used to calculate the segment angle $(\zeta)$ based on the pixel point pitch $(\Lambda)$ at time step $(3 \times \Delta t)$. (d) The segment angle can be determined at each time step to emulate a calibration curve for data fitting purposes.

The dwell and step model equations are

$$
\begin{aligned}
& \frac{\mathrm{d} x^{\prime}}{\mathrm{d} t}=\left[v_{n} \mathrm{e}^{-\left(x^{\prime}\right)^{2} / 2 \sigma_{b}^{2}}\left[\left(1-K_{1}\right)+K_{1} \mathrm{e}^{-K_{2} t}\right]\right]\left[\frac{\frac{\mathrm{d} z}{\mathrm{~d} x^{\prime}}}{\sqrt{1+\left(\frac{\mathrm{d} z}{\mathrm{~d} x^{\prime}}\right)^{2}}}\right] \\
& \frac{\mathrm{d} z}{\mathrm{~d} t}=\left[v_{n} \mathrm{e}^{-\left(x^{\prime}\right)^{2} / 2 \sigma_{b}^{2}}\left[\left(1-K_{1}\right)+K_{1} \mathrm{e}^{-K_{2} t}\right]\right]\left[\frac{1}{\sqrt{1+\left(\frac{\mathrm{d} z}{\mathrm{~d} x^{\prime}}\right)^{2}}}\right]
\end{aligned}
$$

Each term will be discussed independently. The mathematical expression residing in the first set of square brackets in eqs 14 and 15 represents the magnitude of the growth velocity normal to the surface $s\left(x^{\prime}, z\right)$ at position $\left(x^{\prime}\right)$. The maximum possible growth velocity is $\left(v_{n}\right)$, and it occurs at $x^{\prime}=0$ and serves as a fitting parameter. FEBID dependence on the primary electron Gaussian beam profile is captured through the exponential term

$$
\mathrm{e}^{-\left(x^{\prime}\right)^{2} / 2 \sigma_{b}^{2}}
$$

where $\left(\sigma_{b}\right)$ is the primary electron beam radius standard deviation. Isotropic SE emission leads to surface growth in $\left(x^{\prime}\right)$ as well as $(z)$. As a result, the trigonometric terms in the second set of square brackets in eqs 14 and 15 partition the growth velocity into $\left(x^{\prime}\right)$ and $(z)$ components along the surface normal [S10: Trigonometric functions in the simple FEBID model].

Time-dependent precursor surface depletion is captured by the term

$$
\left(1-K_{1}\right)+K_{1} \mathrm{e}^{-K_{2} t}
$$

A maximum fractional reduction in coverage is introduced $\left(K_{1}\right)$ and spans a range from $0-1$. An exponential reduction in precursor coverage in time is driven by the frequency factor $\left(K_{2}\right)$. Precursor coverage saturates to $\left(1-K_{1}\right)$ following complete decay $(t \rightarrow \infty)$ from an initial value of 1 . This term is intended to emulate precursor depletion during FEBID inside the (BIR) without explicit treatment of the source of depletion. Fitting parameters include (1) $v_{n}$, (2) $K_{1}$, and (3) $K_{2}$ in the program user interface as well as (4) the initial nuclei radius $\left(r_{N}\right)$ as defined in Figure 4.

A lower threshold dwell time has been observed for segment nucleation (see Figure 4 (model) and for experiments Figure 5a). Beam-deposit intersection is lost below this threshold during beam scanning for segment growth, relegating FEBID to 2D-growth, because the volumetric growth rate at small dwell times does not provide enough deposition for vertical take-off. As a consequence, the beamsubstrate intersection is renewed, and nanowire growth ensues on the substrate. Moreover, the threshold value depends on instrument

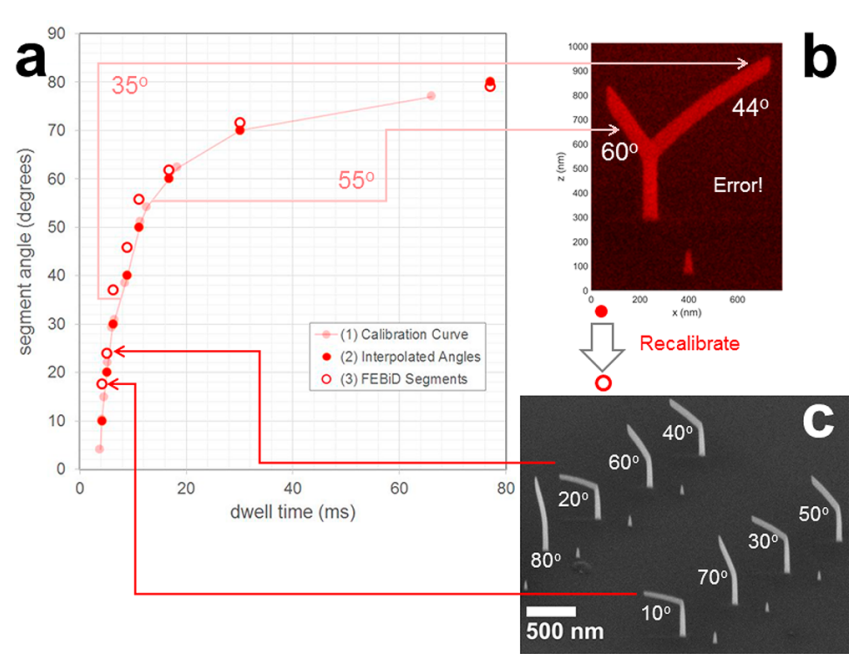

Figure 5. (a) Calibration curve derived at position GIS_1 (light red curve) created using a primary electron energy of $30 \mathrm{keV}$ and a beam current of $31 \mathrm{pA}$. (b) A FEBID branch structure grown at the position GIS_2 but using the calibration curve derived at position GIS_1. The segment angles on exposure level \#2 are incorrect. (c) A total of eight new pillar + segment structures were deposited to create a new calibration curve at position GIS 2 but using the calibration curve from position GIS_1. Interpolation of the experimental calibration curve was used to create the exposure files for the segments spanning the segment angle range of $10-80^{\circ}$, in steps of $10^{\circ}$ (dark red and filled data points). The segment angles are shown as the red ring data points. Conveniently, the calibration curve changes by no more than $10^{\circ}$ over all segment angles deposited for a relatively large displacement in GIS position. Thus, it is usually possible to calibrate the program with a single iteration of recalibration for standard FEBID conditions. 
settings (Table 2). This phenomenon is captured in the model by the introduction of a pre-existing semicircular arc, or nuclei, on the substrate. This model leads to the following initial conditions

$$
\begin{aligned}
& x^{\prime}(t=0)=r_{N} \cos \theta \\
& z(t=0)=\sqrt{r_{N}^{2}-\left(x^{\prime}\right)^{2}}
\end{aligned}
$$

where the surface is discretized into unique steps using the vector of $\left(x^{\prime}\right)$ values (Figure 4a). This assumption is purely empirical but captures well the variation in the minimum dwell time required for segment take-off, which has been observed to vary with experimental set-up.

5.2. Numerical Evolution of the FEBID Model. Eqs 14 and 15 are solved numerically. The total dwell time spanning the calibration curve (typically $<100 \mathrm{~ms}$ ) is evolved in discrete time increments, typically $<1 \mathrm{~ms}$. Starting with a circular nuclei of radius $\left(r_{N}\right)$, spanning $\theta=0$ to $90^{\circ}$, the shape is discretized into a series of arcs of length $\Delta s$. The growth of the nucleus proceeds per time step $(\Delta t)$ as follows (Figure 4)

1. The slope of the nuclei surface is estimated $\left(\mathrm{d} z / \mathrm{d} x^{\prime}\right)$ at each point on the surface.

2. The complementary surface normal vectors are computed from the vector of $\left(\mathrm{d} z / \mathrm{d} x^{\prime}\right)$ values.

3. The nuclei surface is evolved in $\left(x^{\prime}\right)$ and $(z)$ according to eqs 14 and 15 as

$$
\begin{aligned}
& x_{q+1}^{\prime}=x_{q}^{\prime}+\frac{\mathrm{d} x^{\prime}}{\mathrm{d} t} \Delta t \\
& z_{q+1}=z_{q}+\frac{\mathrm{d} z}{\mathrm{~d} t} \Delta t
\end{aligned}
$$

4. The surface is rediscretized into $\left(x^{\prime}, z\right)$ nodes, keeping the surface node spacing $(\Delta s)$ as close to the original $\left(\Delta s_{\mathrm{o}}\right)$ as possible.

5. The segment angle $(\zeta)$ is estimated during each time step

$$
t_{q+1}=t_{q}+\Delta t
$$

6. A full calibration curve is estimated by repeating steps $1-6$ until the total dwell time $\left(t_{q+1}\right)$ equals, or exceeds, the maximum dwell time in the calibration data.

7. The calibration curve fit is evaluated based on the rules described in [S11: Calibration curve fitting rules].

The step-by-step procedure to execute a calibration data fit using 3BID is provided in Method \#3 (video and tutorial).

\section{DISCUSSION}

In order to illustrate the full power of the developed CAD software, we will now discuss a series of real-world problems, which all 3D-FEBID users may encounter. In what follows, we will discuss five cases of practical interest:

1. System recalibration due to GIS displacement.

2. Using a continuous versus an intermittent exposure.

3. Precursor depletion in the diffusion enhanced regime.

4. Fitting of calibrated data.

5. Compensation of segment bending.

6.1. Practical 3D-FEBID Exposure and Calibration. A practical example of CAD-based 3D-FEBID is now presented and discussed, which is expected to arise for users of the program. Consider the following situation where the GIS position has been changed relative to the electron beam impact position on the substrate surface in preparation for 3D-FEBID.

A calibration curve has been previously generated for a GIS position of $\Delta x=220 \mu \mathrm{m}, \Delta y=220 \mu \mathrm{m}$, and $\Delta z=50 \mu \mathrm{m}$ for the precursor $\mathrm{MeCp}\left(\mathrm{Pt}^{\mathrm{IV}}\right) \mathrm{Me}_{3}$. The reservoir temperature of the crucible is always set to $45{ }^{\circ} \mathrm{C}$. This GIS position will be called GIS 1 below. The beam parameters for FEBID consisted of a primary electron energy and a beam current of $30 \mathrm{keV}$ and $31 \mathrm{pA}$, respectively. The imaging focal plane was located $\sim 50$ $\mathrm{nm}$ above the substrate plane. The resulting mean vertical growth rate was found to be $134 \pm 5 \mathrm{~nm} / \mathrm{s}$ during the FEBID experiments.

The following example was engineered to answer the question "What is the difference in pillar growth rate and segment angle if the old calibration curve is used to create the exposure file for FEBID at the new GIS position?" For this purpose, now consider the situation where the GIS is moved to a new position: $\Delta x=30 \mu \mathrm{m}, \Delta y=30 \mu \mathrm{m}$, and $\Delta z=100 \mu \mathrm{m}$. The angle of the GIS nozzle remains fixed at $38^{\circ}$ with respect to the substrate surface normal.

Figure 5a shows the calibration curve for position GIS 1, as the light red semitransparent plot including round experimental data points. First, the $\mathrm{CAD}$ program is used to create the exposure file required for the FEBID of the branch nanostructure at GIS_2, using the calibration determined at GIS_1. A pillar element was defined on exposure level \#1 with a length of $300 \mathrm{~nm}$ to exclude influence from the substrate. ${ }^{51}$ Exposure level \#2 was defined with two segments, segment ( $i=$ $2, j=1$ ) (see figure with a total length of $600 \mathrm{~nm}$ and $\zeta_{21}=35^{\circ}$ and segment $(i=2, j=2)$, which was specified to be $300 \mathrm{~nm}$ long with a $\zeta_{22}=55^{\circ}$. The FEBID results at GIS_2 are shown in Figure $5 \mathrm{~b}$, where the substrate was tilted at $52^{-}$with respect to the substrate surface normal during electron imaging.

The branch deposited at GIS_2 (Figure 5b) shows the expected variation in critical dimensions relative to the $\mathrm{CAD}$, due to the lack of an appropriate calibration at GIS 2. The vertical growth rate at GIS_2 was found to be $170 \pm \overline{5} \mathrm{~nm} / \mathrm{s}$. The segment angles were measured as $\zeta_{21}=44 \pm 2^{\circ}$ and $\zeta_{22}=$ $60 \pm 2^{\circ}$. Evidently, the precursor flux is larger at GIS_2 leading to a pillar that is taller than the design by a factor of $\sim 1.3$, whereas the segment \#1 angle was $\Delta \zeta_{21}=+9^{\circ}$ (factor of $\sim 1.3$ ), and the segment $\# 2$ angle was $\Delta \zeta_{22}=+5^{\circ}$ (factor of $\sim 1.1$ ) relative to the CAD. GIS simulations were in agreement with this experimentally observed change (Figures S3 and S4).

The relatively small variation in segment angle between $\mathrm{CAD}$ and FEBID experiments made it possible to create a new calibration curve at GIS 2, using a single iteration of pillar + segment deposition (Figure 5c) based on the calibration curve at GIS_1. For example, a total of eight new pillar + segment elements were deposited covering the segment angle range from $10-80^{\circ}$ in steps of $10^{\circ}$. The resulting segment angles were measured and plotted as shown in Figure 5a as the dark, red ring data points. This data represents the updated calibration curve at position GIS_2.

This example provides a useful guide for the expected variation in 3D-FEBID for a common GIS realignment-GIS displacement on the order of hundreds for microns under standard delivery conditions for the precursor $\mathrm{MeCp}\left(\mathrm{Pt}^{\mathrm{IV}}\right) \mathrm{Me}_{3}$ yields segment angle error on the order of a few degrees.

6.2. Implications of Continuous versus Intermittent Exposure. Continuous segment exposure drives the 3DFEBID regime toward the precursor-limited regime. Precursorlimited conditions can develop not only from mass transport limitations, such as a relatively low precursor impingement flux and/or surface diffusion flux but also can be precursor desorption limited. ${ }^{53}$ For example, in the branch geometry example (reproduced in Figure 6a), the segment $(i=2, j=2$ ) was removed from the branch FEBID exposure pattern, 


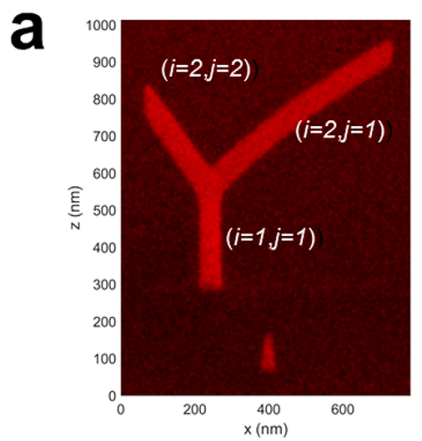

C

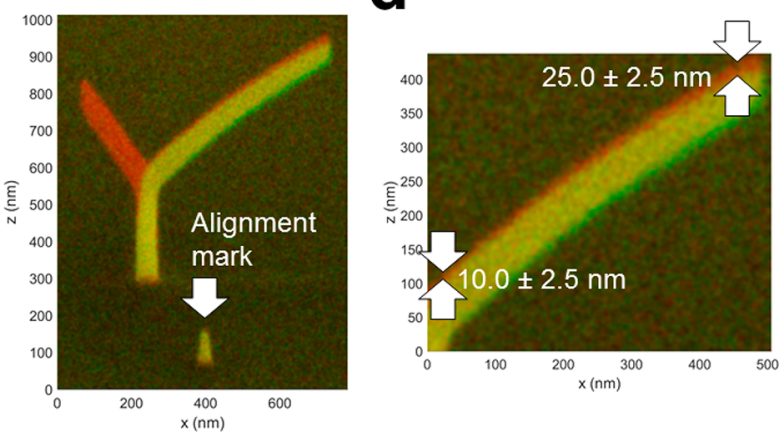

Figure 6. (a) FEBID $\mathrm{PtC}_{x}$ branch grown at $30 \mathrm{keV} / 31 \mathrm{pA}$ using the precursor $\mathrm{MeCp}\left(\mathrm{Pt}^{\mathrm{IV}}\right) \mathrm{Me}_{3}$. The initial CAD-specified pillar height on exposure level \#1 was $300 \mathrm{~nm}$. The relatively longer segment on exposure level \#2 (right segment) was specified as $35^{\circ}$ with a segment length of $600 \mathrm{~nm}$. The resulting segment angle measures at $\zeta=44 \pm$ $2^{\circ}$. The shorter segment on exposure level \#2 (left branch) was specified as $55^{\circ}$ and $300 \mathrm{~nm}$ long. The resulting segment angle measures at $\zeta=60 \pm 2^{\circ}$. (b) A complementary FEBID structure consisting of the right segment only $\left(\zeta=35^{\circ}\right)$ fabricated via continuous exposure. (c) SEM images (a) and (b) were overlapped, with aide of the alignment mark, in order to reveal the result of intermittent (a) versus continuous (b) exposure for the relatively long segment. (d) The magnified view of (c) shows the deviation in segment angle due to the patterning-induced additional refresh time by intermittent exposure-the growth rate slightly increases, because the precursor coverage at the tip of the growing segment is slightly higher during intermittent exposure (equivalent to a slight DER $\rightarrow$ ELR shift).

converting exposure level \#2 into a continuous exposure (Figure 6b). A common alignment mark was used to overlay the images in $(\mathrm{r}, \mathrm{g}, \mathrm{b})$ space (Figure $6 \mathrm{c}$ ) to directly evaluate the implications of exposure mode on the segment angle: red = intermittent $/$ continuous, green = purely continuous, and yellow = overlay. Magnification of the overlay image (Figure 6d) shows several important consequences of exposure sequencing as well as the general 3D-FEBID CAD approach reported here

- Segment angle depends on the exposure sequence accumulating tens of nanometers of difference per hundreds of nanometers of segment growth.

- The linear segment approximation inherently assumed in the $\mathrm{CAD}$ program is generally invalid for segment lengths beyond $\sim 0.5 \mu \mathrm{m}$. Compensation in the CAD design may be required for 3D-mesh objects with segments approaching this limit.

- Precursor depletion at the BIR located at the tip of the segment primarily causes the observed differences in exposure mode (see the next section).

6.3. Diffusion Enhanced Regime Conditions. The role of precursor depletion can be extracted from 3D-FEBID simulations of the branch deposit. A subtle downward deflection occurs in segment growth when intermittent exposure transitions to the continuous mode (Figure 7a) (see
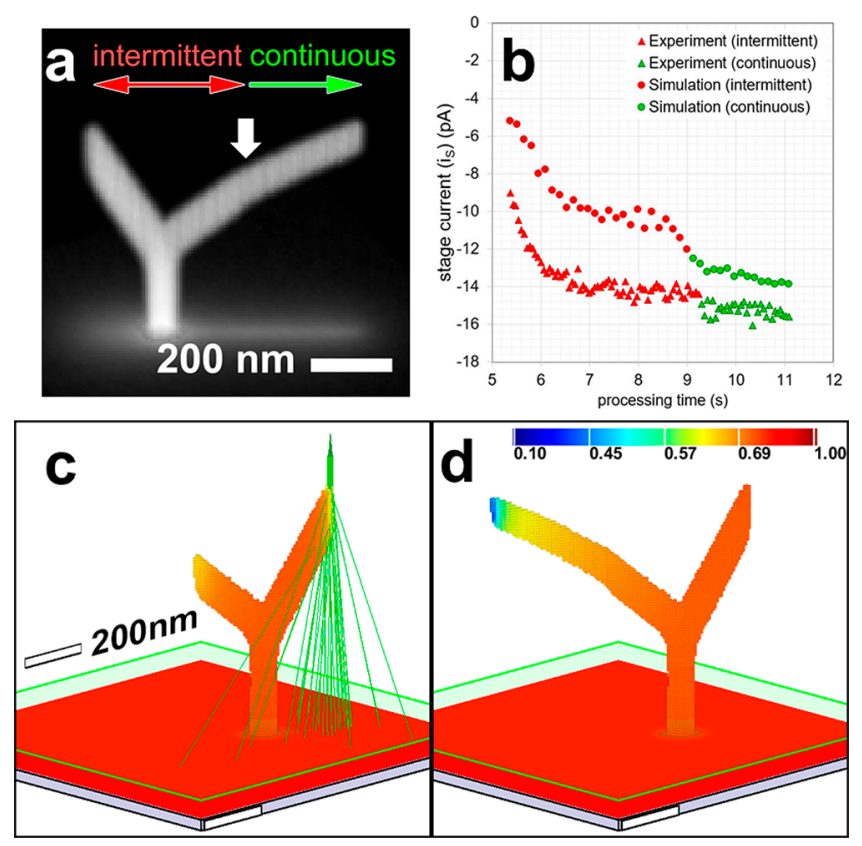

Figure 7. (a) Virtual SEM image of the simulation results for a FEBID $\mathrm{PtC}_{5}$ branch structure grown using $30 \mathrm{keV} / 31 \mathrm{pA}$ using the precursor $\mathrm{MeCp}\left(\mathrm{Pt}^{\mathrm{IV}}\right) \mathrm{Me}_{3}$. The arrow indicates when the level \#2 exposure transitions from an intermittent to continuous exposure. (b) The stage current collected during FEBID of exposure level \#2. Experimental (triangles) and simulated (circles) data are both shown. In this data channel, the transition from intermittent (red) to continuous (green) exposure is seen as a drop in current. (c) A 3D-image of the surface voxels surrounding the deposit with the fourth dimension indicating fraction surface coverage of $\mathrm{MeCp}\left(\mathrm{Pt}^{\mathrm{IV}}\right) \mathrm{Me}_{3}$. This image frame was captured at the moment the deposition of segment $(i=2, j=2)$ is completed, just before continuous exposure ensues for the completion of segment $(i=2, j=1)$. Select electron trajectories are shown for visual reference. (d) The precursor surface coverage at the instant segment $(i=2, j=1)$ is completed. A precursor coverage gradient exists along the para-axial dimension of the segment. The color scale is the fraction of surface covered by precursor.

the details of the exposure sequencing scheme in Figure 3). The 3D-FEBID simulation was conducted at the position GIS_2, making it complementary to the experimental example presented in Figure 6. Work is currently underway to evaluate simulation replication of time-dependent stage current data collected during experiments. In particular, the gradual but downward curving nature of segments grown in continuous mode, e.g, Figure $6 \mathrm{~b}$, will be related to precursor desorption in a future publication. Initial results related to the current problem are now presented.

Figure $7 \mathrm{~b}$ shows stage current data collected during the FEBID of exposure level \#2 and during a simulation. In both cases, a decrease in stage current is observed which correlates with the change in exposure mode from intermittent to continuous. Data points have been color-coded to clearly mark the exposure transition-red data was collected during intermittent exposure, while green data was collected during the continuous mode. Figure $7 \mathrm{c}$ shows the precursor surface coverage at the completion of the final beam dwell on the segment $(i=2, j=2)$. The precursor gradient adjacent to the 
BIR is indicative of precursor-limited conditions. Equally as important, precursor depletion is still evident on the segment $(i$ $=2, j=1$ ) (see the yellow to red gradient extending down the segment axis). Precursor coverage has not had enough time to reach the equilibrium value (red) during the natural refresh time imposed by intermittent mode.

The abrupt decrease in the stage current at the intermittent/ continuous transition is a characteristic of a slightly reduced segment angle, which also can be seen in the simulation images in Figure $7 \mathrm{a}$,d. It was found that a reduction in average coverage in the BIR was responsible for the observed reduction in segment angle.

The precursor surface coverage in the BIR was calculated, after the conclusion of beam dwell at each pixel point, by taking the average surface coverage for surface voxels located in the BIR. These values were then further averaged over the various exposure modes. Averaging over the continuous exposure pillar growth phase yielded a fractional coverage of $0.63 \pm 0.02$. Precursor surface coverage is relatively high during this phase, because the base of the pillar is in contact with the large precursor reservoir available on the substrate surface. However, because the pillar growth phase is continuous, the beam constantly irradiates a significant portion of the pillar surface (1), causing a gradient in coverage from the top of the pillar to the bottom.

The fractional precursor coverage reduced to $0.56 \pm 0.03$ during intermittent exposure. The reduction in coverage occurs, because the segment is irradiated by both the incoming and outgoing primary electron beam at the suspended BIR, while the distance from the substrate precursor reservoir to the BIR has increased. Moreover, beam grazing at the growing segment face further depletes the precursor while aiding in lateral growth. However, intermittent exposure between the segments on exposure level \#2 introduces a patterning-induced, precursor refresh time due to a short-range surface diffusion at the edge of the BIR and also from direct gas phase replenishment. This is not the case during the last phase of purely continuous growth on exposure level $\# 2$, where the mean fractional coverage further reduces to $0.40 \pm 0.01$.

Thus, the change in segment angle on transitioning from the intermittent to continuous exposure mode can be directly related to the precursor surface coverage at the segment BIR. In terms of the working regime, this indicates a further shift away from ELR toward DER conditions. The relatively extreme depletion at the segment tip (blue color) is clear in Figure $7 \mathrm{~d}$.

The basic features of the $\mathrm{CAD}$ approach have now been described along with the translation to experiments. The following method shows that more complex designs can be created outside the GUI and later imported into the environment using text files containing vertex and segment lists. In concert with an autosegment detection tool provided in the program (Method \#4 (video and tutorial)), more complex structures can be quickly translated into FEBID exposure files.

6.4. Calibration Curve Fitting. The calibration curve fitting at GIS 1 also suggests precursor coverage as a limiting factor for segment growth. The range of values explored for the data fitting, per parameter, were $(1) v_{\mathrm{nb}}=120: 2: 170[\mathrm{~nm} / \mathrm{s}]$, (2) $K_{1}=0.2: 0.1: 0.8$, and (3) $K_{2}=1 /(4: 2: 36)[1 / \mathrm{ms}]$, and the initial nuclei radius $r_{N}=0.5: 0.025: 0.6[\mathrm{~nm}]$ (notation $\rightarrow$ minimum:increment:maximum). The best fit was based on the fitting criteria presented in [S11: Calibration curve fitting rules] and was found to be

$$
\begin{aligned}
& v_{n}=130 \frac{\mathrm{nm}}{\mathrm{s}} \\
& K_{1}=0.60 \\
& K_{2}=\frac{1}{28} \frac{1}{\mathrm{~ms}} \\
& r_{N}=0.55 \mathrm{~nm}
\end{aligned}
$$

Figure 8a shows again the GIS 1 calibration data along with the best data fit. The maximum vertical growth rate $\left(v_{n}\right)$
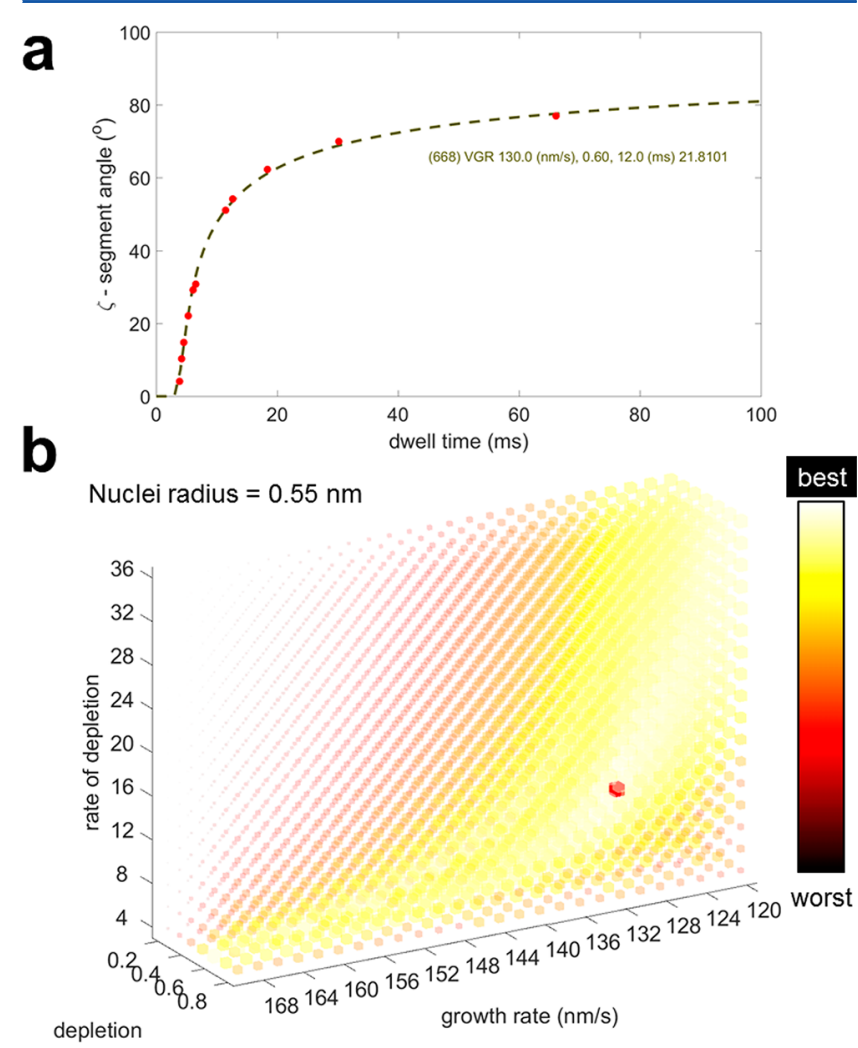

Figure 8. (a) Best calibration curve fit for the range $v_{n}=120: 2: 170$ $[\mathrm{nm} / \mathrm{s}], K_{1}=0.2: 0.1: 0.8, K_{2}=1 / 4: 2: 36[\mathrm{~ms}]$, and $r_{N}=0.5: 0.025: 0.6$ [nm]. (b) Each voxel represents a unique value of $\left(K_{1}, K_{2}\right.$, and $\left.v_{n}\right)$ for $r_{N}=0.55[\mathrm{~nm}]$. Opacity and voxel size increase with the quality of fit. Thus, the white intense strip shows the cluster of best solutions. The very best solution is shown as the bright red voxel and represents the fit shown in (a). The colormap is a logarithmic base 10 scale.

matches within $\sim 3 \%$ of the experimentally measured average value. Please note, the fitting model provides, the fitting model provides only an empirical representation of FEBID, but the FEBID-like character should make it easier for a program user to define a range of fitting parameters based on experimental observations.

Finally, the uniqueness of the solution space can be seen in Figure $8 \mathrm{~b}$. Higher quality solutions are indicated by color (see the colormap), cubic voxel edge length (larger size, better fit), and are more opaque. This approach makes it possible to interpret the 4D-plot of information visually in $3 \mathrm{D}$, where the bright white regions show the best solutions. A 3D-map such as that shown in Figure $8 \mathrm{~b}$ is generated for each nuclei radius. The very best fit solution is shown as the bright red voxel for clarity.

6.5. Low Angle Segment Compensation. Segments are prone to a sublinear bending (Figure $6 \mathrm{~b}$ ). Please note that this 
observation appears to be a general trend under precursorlimited conditions, where the angle $\zeta$ at which bending occurs seems correlated with the segment length, precursor type (see $\mathrm{Me}_{2} \mathrm{Au}(\mathrm{acac})$ case in ${ }^{51}$ ), precursor impingement rate, beam size, beam voltage, etc. Nonetheless, a compensation tactic has been provided with the program (Method \#5 (video and tutorial)) to compensate for this phenomen.

The first step of compensation requires the subdivision of all mesh elements, i.e., pillar and segments, of the 3D-mesh object. A new vertex is introduced at the midpoint of each element during a single subdivision step. As a result, the number of total segments is doubled for each subdivision. Segments are automatically reassigned, and the order of exposure is preserved as defined before subdivision.

Segment compensation is performed on a per segment basis. A mathematical function is used to impose a superlinear compensation in the $\mathrm{CAD}$. The key variable in the function defines a rate of change of segment angle as a function of distance along the segment $\left(\mathrm{d} \zeta_{i j} / \mathrm{d} x^{\prime}\right)$. The function is now discussed in the segment frame of reference $\left(x^{\prime}, z\right)$.

The segment angle $\left(\zeta_{i j}\right)$ is calculated using the initial and final vertices that defined the original segment or pillar of interest, prior to any subdivision operation. This value serves as the initial value $\left(x^{\prime}=0\right)$ in the mathematical function, which is

$$
\zeta_{i j}\left(x^{\prime}\right)=\zeta_{i j}\left(x^{\prime}=0\right)+\frac{\mathrm{d} \zeta_{i j}}{\mathrm{~d} x^{\prime}} x^{\prime}
$$

The $z$-coordinate of any vertex that is found to lie along the line spanning the initial and final vertex is increased according to

$$
z=z_{o}+x^{\prime} \tan \left(\zeta_{i j}\left(x^{\prime}\right)\right)
$$

and a new vertex is created with that $(z)$ position. Segment definition is transferred to the newly created vertices.

Figure 9 shows the results of segment angle compensation for the branch nanostructure. The digital nature of segment discretization was evident when fewer subdivisions were used (data not shown). It was found that three subdivision operations were required to produce smoothly curving segments. For clarity, Figure 9a shows the branch $\mathrm{CAD}$ following two subdivision operations and segment compensation. The compensation was applied to the linear segment originally spanning the vertices with indices of $u_{i}=1$ to $u_{f}=2$ and $\zeta=30^{\circ}$. Following the compensation operation, vertices 2 , 6,10 , and 11 were replicated and shifted using eqs 18 and 19 yielding the new, compensated set of vertices with indices 1417 (Figure 9a).

FEBID experiments were conducted for a range of segment compensation values

$$
\frac{\mathrm{d} \zeta}{\mathrm{d} x^{\prime}}=(0,0.010,0.012,0.014,0.016)\left[\frac{\text { degrees }}{\mathrm{nm}}\right]
$$

using the GIS 2 position. Figure 9b shows the secant line approximation of $\zeta$ derived from SEM images taken at $52^{\circ}$ substrate tilt. The branch with no segment compensation (orange data line) exhibits the sublinear bending characteristic of low-angle segments. Further, this branch (Figure 9c) yields an average segment angle of $24^{\circ}$, which is less than the CAD specification of $30^{\circ}$. This is a typical variation observed in the translation from calibration curve to FEBID exposure due to the steep slope of the calibration curve in the low-angle range. In other words, this experiment was conducted without
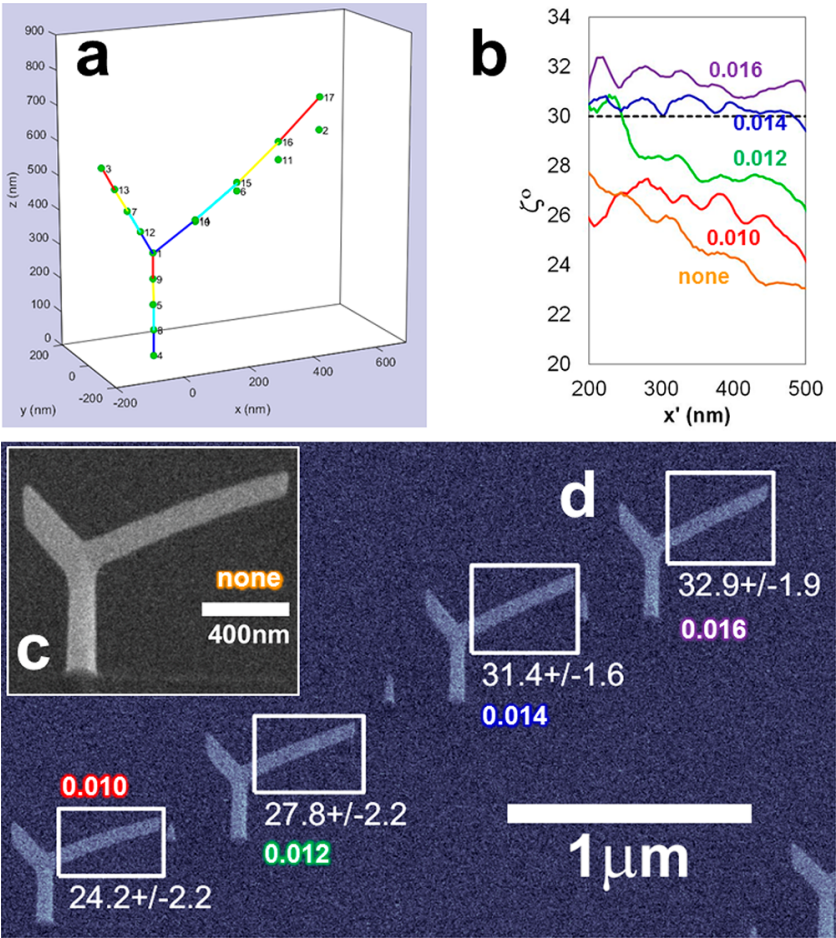

Figure 9. (a) Segment angle compensation applied to the segment spanning vertices $1-2\left(L=600 \mathrm{~nm}, \zeta=30^{\circ}\right)$. (b) Segment angle (secant approximation) vs $L_{x y}$ for angle compensation executed at $\mathrm{d} \zeta$ / $\mathrm{d} x^{\prime}=0.010,0.012,0.014$, and $0.016[\% / \mathrm{nm}]$. (c) The CAD-specified $\zeta$ $=30^{\circ}$, yet the final deposit exhibited sublinear growth with a continuously decreasing segment angle as a function of $x^{\prime}$. Also, see "none" in (b). (d) The best reproduction of design was achieved with $0.014[\% / \mathrm{nm}]$. The mean segment angle for each segment is shown. Electron imaging was conducted at $52^{\circ}$. A spatial autocorrelation analysis was used to compute the segment angles directly from the tilted SEM images shown in (c) and (d).

recalibration. $\mathrm{d} \zeta_{i j} / \mathrm{d} x^{\prime}$ values of $0.010\left[{ }^{\circ} / \mathrm{nm}\right]$ (red line) and $0.012[\% / \mathrm{nm}]$ (green line) both (1) increased the average segment angle along $\left(x^{\prime}\right)$ and (2) reduced the segment bending. The segment angle variation of $\mathrm{d} \zeta_{i j} / \mathrm{d} x^{\prime}=0.014[\%$ $\mathrm{nm}$ ] proved to be the best correction value (blue line), minimizing the bending while also yielding a mean angle $(31.4$ $\pm 1.6^{\circ}$ ) close to the target value of $30^{\circ}$ (black, dashed line). The optimized structure is shown in Figure $9 \mathrm{~d}$.

Examples of both an ideal and nonideal design for FEBID will now be discussed to highlight the advantages/limitations of the $\mathrm{CAD}$ program. An ideal $\mathrm{CAD}$ structure is the regular icosahedron shown in Figure 10a, which has a small fill factorthe polyhedra, with interconnecting faces and a hollow core, is an ideal geometry. Moreover, segment elements have been selectively removed from the regular icosahedron with the intended purpose of minimizing primary electron beam exposure beyond the beam impact region (BIR). Only along an imaginary vertical line, extending through vertices 9,12 , and 13, will overexposure occur. Nonetheless, overexposure due to converging segments at vertex \#9 was avoided using the proximity correction tool described in (Method \#4, Figures 16-17) where $s \_i=1 \mathrm{~nm}$ and $s \_f=4 \mathrm{~nm}$. As a result, the array of regular icosahedra shown in Figure $10 \mathrm{~b}$ reproduced the intended design. Please see Method \#6: Array exposure (video and tutorial) for instructions on how to create a $2 \mathrm{D}$-array of replicate $3 \mathrm{D}$-objects projected in the focal plane. 


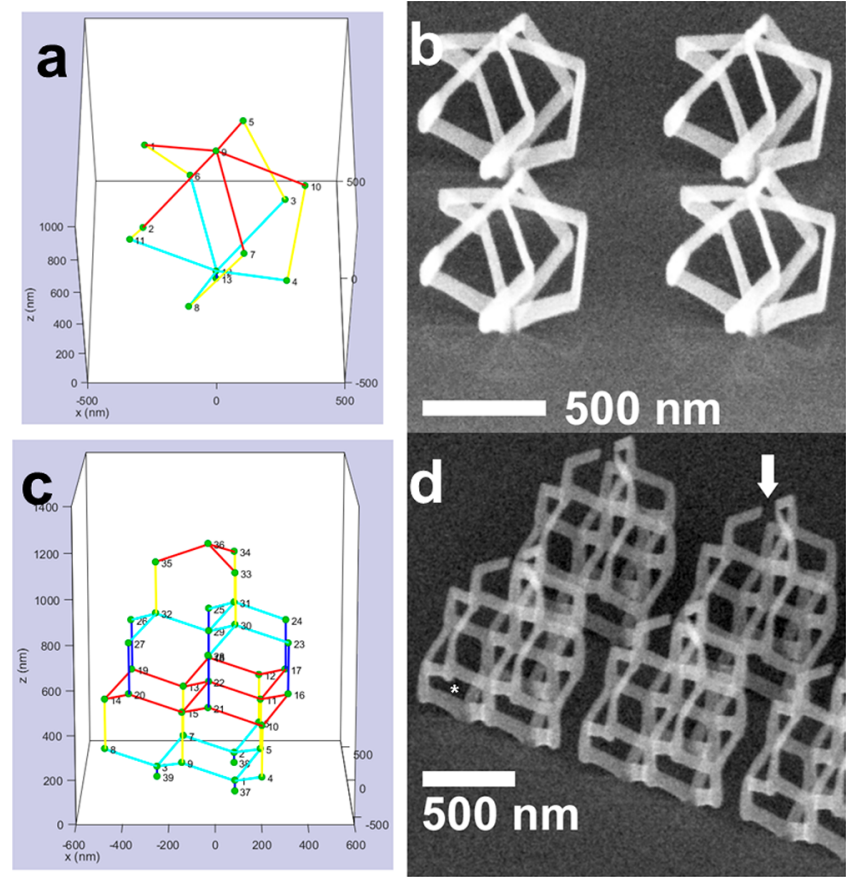

Figure 10. (a) An icosahedron 3D-mesh object design containing four exposure levels-level \#1 (blue), \#2 (light blue), \#3 (yellow), and \#4 (red). The intermittent exposure layout was used. The design is shown at a virtual substrate tilt of $52^{\circ}$. (b) The complementary FEBID exposure of the design in (a) but for replication of the object in a square, $2 \mathrm{D}$-array with a spacing of $1 \mu \mathrm{m}$. The substrate tilt is $52^{\circ}$ and complementary to the design image. (c) A diamond lattice 3D-mesh object design with eight exposure levels, also with an intermittent exposure layout. For clarity, the virtual substrate tilt is $15^{\circ}$ to better reveal the design, which differs from the tilt angle of $52^{\circ}$ shown for the complementary experiments in (d). The diamond lattice contains alternating cycles of pillar growth and low segment angle exposure $(\zeta$ $=19.5^{\circ}$ - challenging conditions for FEBID as shown by the superimposed arrow showing that the final structure failed to close completely at the apex terminus.

Conversely, an example of a challenging 3D-object for FEBID replication is the diamond lattice structure (lattice parameter $=250 \mathrm{~nm}$ ) shown in Figure 10c. The design consists of alternating exposure levels of pillar elements $\left(\zeta=90^{\circ}\right)$ followed by low-angle segments $\left(\zeta=19.5^{\circ}\right)$. The suspended pillar structure is prone to overexposure on the underside of the suspended pillar (Figure 10d, see *). Further, the prolonged, stationary dwell time required for pillar elements can lead to the thickening of surrounding elements due to elastic forward and backscattering of primary electrons. The low-angle segment introduces another source of error for FEBID. The error per FEBID exposure of low-angle segments is relatively large, because the calibration curve (segment angle versus primary electron beam dwell time) is steep in this range (see Figure 5a). The alternating cycles of pillar exposure and low-angle segment exposure accumulate error due to the layer-by-layer deposition required for $3 \mathrm{D}$-nanoprinting. As a result, the final three segments failed to converge at the apex of the deposit (Figure $10 \mathrm{~d}$, see $\rightarrow$ ). In addition, segment bowing/bending impacts convergence as well.

\section{CONCLUSIONS}

The CAD program (3BID) makes it possible to quickly design 3D-mesh objects for FEBID and export exposure files for direct use with an electron microscope equipped with a gas-injection system and the possibility of programmable beam translation. The conventional methodology of FEBID by trial and error is replaced with a more rigorous approach implementing a CAD environment that is calibrated for FEBID. The program has been optimized for 3D-mesh style objects with sizes on the order of hundreds of nanometers up to several micrometers. Ideally, the 3D-object should have a low volumetric fill factor with a design that avoids multiple intersections of the primary beam with the deposit. This minimizes unwanted deposition preserving the fidelity of the original design.

$3 \mathrm{D}$-objects with an average element length ranging from 200-600 nm are recommended for exposure. These approximate limits apply to the conditions demonstrated here using a primary electron energy of $30 \mathrm{keV}$ and primary beam current $<100 \mathrm{pA}$. The elastic scattering interaction volume proximity effect yields detrimental deposition outside the BIR for an average element spacing below roughly $200 \mathrm{~nm}$, while segment bending will occur for relatively long segments $(>600 \mathrm{~nm})$. A segment angle correction is included in the $\mathrm{CAD}$ program to create longer segments.

The program is also recommended for use as a conversion tool. In other words, vertex and segment lists can be defined outside the $\mathrm{CAD}$ environment and imported into the program. This capability, along with autosegment detection features, makes it possible to quickly import and define a 3D-object that can then be edited in the $\mathrm{CAD}$ environment. For further demonstrations of 3D-FEBID, see refs 49,50, 52, and 54-56, where nanostructures were created, exported, and exposed using the FEBID CAD program.

\section{ASSOCIATED CONTENT}

\section{S Supporting Information}

The Supporting Information is available free of charge on the ACS Publications website at DOI: 10.1021/acsanm.7b00342.

As the presented work has been done in very much detail, we provide supplements on (1) how pillar versus segment exposure element is selected for a FEBID exposure file; (2) how exposure files are created when a microscope has a maximum allowable dwell time; (3) a simple dwell time scaling for FEBID exposure files; (4) experiments demonstrating the time-dependent vertical growth rate for FEBID using the precursor $\mathrm{MeCpP}$ $\mathrm{t}^{\mathrm{IV}} \mathrm{Me}_{3}$; (5) a list of key experimental parameters influencing 3D-FEBID; (6) the current maximum electron beam dwell time in the FEBID CAD (3BID) program; (7) CAD program calibration data interpolation and extrapolation; (8) file list for the FEBID CAD (3BID) program before and after execution as well as a list of GUI topics covered in each video method; (9) the $\left(x^{\prime}, z\right)$ coordinate system in the frame of reference of an exposure "segment"; (10) a derivation of the simple FEBID model used to fit calibration curve data in the program; (11) calibration curve fitting rules; and (12) the local precursor substrate surface pressure for two different gas-injection system (GIS) positions (PDF) Method tutorials: (1) basic CAD use; (2) calibration file preparation; (3) calibration data fit; (4) autosegment detection; (5) segment compensation; and (6) array exposure (ZIP)

Method videos: (1) simple pillar and segment; (2) calibration file preparation; (3) calibration data fit; (4) 
autosegment detection; (5) segment compensation; (6) array exposure; and (7) NanoBranchBasic CAD (ZIP) EBiD 3D (CAD) program files (ZIP)

\section{AUTHOR INFORMATION}

\section{Corresponding Authors}

*E-mail: fo2@ornl.gov (J.D.F.)

*E-mail: harald.plank@felmi-zfe.at (H.P.)

\section{ORCID}

Jason D. Fowlkes: 0000-0002-6295-5115

A. Fernández-Pacheco: 0000-0002-3862-8472

D. Sanz-Hernández: 0000-0002-5552-8836

Michael G. Stanford: 0000-0001-9663-1138

H. Plank: 0000-0003-1112-0908

\section{Funding}

This manuscript has been authored by UT-Battelle, LLC under Contract No. DE-AC05-00OR22725 with the U.S. Department of Energy. The United States Government retains and the publisher, by accepting the article for publication, acknowledges that the United States Government retains a nonexclusive, paidup, irrevocable, worldwide license to publish or reproduce the published form of this manuscript, or allow others to do so, for United States Government purposes. The Department of Energy will provide public access to these results of federally sponsored research in accordance with the DOE Public Access Plan (http://energy.gov/downloads/doe-public-access-plan).

\section{Notes}

The authors declare no competing financial interest.

\section{ACKNOWLEDGMENTS}

J.D.F., R.W., P.D.R., A.F.P., and H.P. acknowledge that the creation of the $\mathrm{CAD}$ environment was conducted at the Center for Nanophase Materials Sciences, which is a DOE Office of Science User Facility. R.W. and H.P. gratefully acknowledge the valuable support provided from Prof. Dr. Ferdinand Hofer. R.W. and H.P. also acknowledge financial support by the COST action CELINA (Nr. CM1301), EUROSTARS project TRIPLE-S (Nr. E! 8213), the bmvit exchange program, and FFG-Production of the Future project SENTINEL (Nr. 850652). L.S., D.S.H., and A.F.P. acknowledge funding from EPSRC, grant numbers EP/M008517/1 and EP/L015978/1, and from the Winton Program for the Physics of Sustainability.

\section{REFERENCES}

(1) Lukatskaya, M. R.; Dunn, B.; Gogotsi, Y. Multidimensional Materials and Device Architectures for Future Hybrid Energy Storage. Nat. Commun. 2016, 7, 12647.

(2) Kyeremateng, N. A.; Brousse, T.; Pech, D. Microsupercapacitors as Miniaturized Energy-Storage Components for On-Chip Electronics. Nat. Nanotechnol. 2016, 12, 7-15.

(3) Fernandez-Pacheco, A.; Streubel, R.; Fruchart, O.; Hertel, R; Fischer, P.; Cowburn, R. P. Three-Dimensional Nanomagnetism. Nat. Commun. 2017, 8, 15756.

(4) O’Donnell, J.; Kim, M.; Yoon, H. S. A Review on Electromechanical Devices Fabricated by Additive Manufacturing. J. Manuf. Sci. E-T ASME 2017, 139, 010801.

(5) Hirt, L.; Reiser, A.; Spolenak, R.; Zambelli, T. Additive Manufacturing of Metal Structures at the Micrometer Scale. Adv. Mater. 2017, 29, 1604211.

(6) Engstrom, D. S.; Porter, B.; Pacios, M.; Bhaskaran, H. Additive Nanomanufacturing - A Review. J. Mater. Res. 2014, 29, 1792-1816.
(7) Utke, I., Moshkalev, S., Russell, P., Eds. Nanofabrication Using Focused Ion and Electron Beams: Principles and Applications; Oxford University Press; 2012.

(8) Utke, I.; Hoffmann, P.; Melngailis, J. Gas-Assisted Focused Electron Beam and Ion Beam Processing and Fabrication. J. Vac. Sci. Technol. B 2008, 26, 1197-1276.

(9) Hagen, C. W. The Future of Focused Electron Beam-Induced Processing. Appl. Phys. A: Mater. Sci. Process. 2014, 117, 1599-1605.

(10) Geier, B.; Gspan, C.; Winkler, R.; Schmied, R.; Fowlkes, J. D.; Fitzek, H.; Rauch, S.; Rattenberger, J.; Rack, P. D.; Plank, H. Rapid and Highly Compact Purification for Focused Electron Beam Induced Deposits: A Low Temperature Approach Using Electron Stimulated $\mathrm{H}_{2} \mathrm{O}$ Reactions. J. Phys. Chem. C 2014, 118, 14009-14016.

(11) Plank, H.; Noh, J. H.; Fowlkes, J. D.; Lester, K.; Lewis, B. B.; Rack, P. D. Electron-Beam-Assisted Oxygen Purification at Low Temperatures for Electron-Beam-Induced Pt Deposits: Towards Pure and High-Fidelity Nanostructures. ACS Appl. Mater. Interfaces 2014, 6, 1018-1024.

(12) Belic, D.; Shawrav, M. M.; Gavagnin, M.; Stoger-Pollach, M.; Wanzenboeck, H. D.; Bertagnolli, E. Direct-Write Deposition and Focused-Electron-Beam-Induced Purification of Gold Nanostructures. ACS Appl. Mater. Interfaces 2015, 7, 2467-2479.

(13) Elbadawi, C.; Toth, M.; Lobo, C. J. Pure Platinum Nanostructures Grown by Electron Beam Induced Deposition. ACS Appl. Mater. Interfaces 2013, 5, 9372-9376.

(14) Shawrav, M. M.; Taus, P.; Wanzenboeck, H. D.; Schinnerl, M.; Stoger-Pollach, M.; Schwarz, S.; Steiger-Thirsfeld, A.; Bertagnolli, E. Highly Conductive and Pure Gold Nanostructures Grown by Electron Beam Induced Deposition. Sci. Rep. 2016, 6, 34003.

(15) van Dorp, W. F.; Wu, X.; Mulders, J. J. L.; Harder, S.; Rudolf, P.; De Hosson, J. T. M. Gold Complexes for Focused-Electron-BeamInduced Deposition. Langmuir 2014, 30, 12097-12105.

(16) Warneke, J.; Van Dorp, W. F.; Rudolf, P.; Stano, M.; Papp, P.; Matejcik, S.; Borrmann, T.; Swiderek, P. Acetone and the Precursor Ligand Acetylacetone: Distinctly different Electron Beam Induced Decomposition? Phys. Chem. Chem. Phys. 2015, 17, 1204-1216.

(17) Rosenberg, S. G.; Barclay, M.; Fairbrother, D. H. Electron Induced Reactions of Surface Adsorbed Tungsten Hexacarbonyl (W(CO) . Phys. Chem. Chem. Phys. 2013, 15, 4002-4015.

(18) Rosenberg, S. G.; Barclay, M.; Fairbrother, D. H. Electron Induced Surface Reactions of Organometallic Metal(hfac)(2) Precursors and Deposit Purification. ACS Appl. Mater. Interfaces 2014, 6, 8590-8601.

(19) Spencer, J. A.; Rosenberg, S. G.; Barclay, M.; Wu, Y. C.; McElwee-White, L.; Howard Fairbrother, D. Understanding the Electron-Stimulated Surface Reactions of Organometallic Complexes to Enable Design of Precursors for Electron Beam-Induced Deposition. Appl. Phys. A: Mater. Sci. Process. 2014, 117, 1631-1644.

(20) Wnuk, J. D.; Rosenberg, S. G.; Gorham, J. M.; van Dorp, W. F.; Hagen, C. W.; Fairbrother, D. H. Electron Beam Deposition for Nanofabrication: Insights from Surface Science. Surf. Sci. 2011, 605, 257-266.

(21) Engmann, S.; Stano, M.; Matejcik, S.; Ingolfsson, O. Gas Phase Low Energy Electron Induced Decomposition of the Focused Electron Beam Induced Deposition (FEBID) Precursor Trimethyl (methylcyclopentadienyl) Platinum(IV) ( $\mathrm{MeCpPtMe}_{3}$ ). Phys. Chem. Chem. Phys. 2012, 14, 14611-14618.

(22) Thorman, R. M.; Kumar T. P., R.; Fairbrother, D. H.; Ingolfsson, O. The Role of Low-Energy Electrons in Focused Electron Beam Induced Deposition: Four Case Studies of Representative Precursors. Beilstein J. Nanotechnol. 2015, 6, 1904-1926.

(23) Koops, H. W. P.; Hoinkis, O. E.; Honsberg, M. E. W.; Schmidt, R; Blum, R.; Bottger, G.; Kuligk, A.; Liguda, C.; Eich, M. TwoDimensional Photonic Crystals Produced by Additive Nanolithography with Electron Beam-Induced Deposition Act as Filters in the Infrared. Microelectron. Eng. 2001, 57-58, 995-1001.

(24) Koops, H. W. P.; Kaya, A.; Weber, M. Fabrication and Characterization of Platinum Nanocrystalline Material Grown by 
Electron-Beam Induced Deposition. J. Vac. Sci. Technol., B: Microelectron. Process. Phenom. 1995, 13, 2400-2403.

(25) Koops, H. W. P.; Schossler, C.; Kaya, A.; Weber, M. Conductive Dots, Wires, and Supertips for Field Electron Emitters Produced by Electron-Beam Induced Deposition on Samples having Increased Temperature. J. Vac. Sci. Technol., B: Microelectron. Process. Phenom. 1996, 14, 4105-4109.

(26) Kretz, J.; Rudolph, M.; Weber, M.; Koops, H. W. P. 3Dimensional Structurization by Additive Lithography, Analysis of Deposits Using Tem and Edx, and Application to Field-Emitter Tips. Microelectron. Eng. 1994, 23, 477-481.

(27) Matsui, S.; Kaito, T.; Fujita, J.; Komuro, M.; Kanda, K.; Haruyama, Y. Three-Dimensional Nanostructure Fabrication by Focused-Ion-Beam Chemical Vapor Deposition. J. Vac. Sci. Technol., B: Microelectron. Process. Phenom. 2000, 18, 3181-3184.

(28) Kometani, R.; Warisawa, S.; Ishihara, S. The 3D-Nanostructure Growth Evaluations by the Real-Time Current Monitoring on Focused-Ion-Beam Chemical Vapor Deposition. Microelectron. Eng. 2010, 87, 1044-1048.

(29) Murakami, K.; Takai, M. Characteristics of Nano Electron Source Fabricated using Beam Assisted Process. J. Vac. Sci. Technol., B: Microelectron. Process. Phenom. 2004, 22, 1266-1268.

(30) Hoshino, T.; Watanabe, K.; Kometani, R.; Morita, T.; Kanda, K.; Haruyama, Y.; Kaito, T.; Fujita, J.; Ishida, M.; Ochiai, Y.; Matsui, S. Development of Three-Dimensional Pattern-Generating System for Focused-Ion-Beam Chemical-Vapor Deposition. J. Vac. Sci. Technol., B: Microelectron. Process. Phenom. 2003, 21, 2732-2736.

(31) Bret, T.; Utke, I.; Gaillard, C.; Hoffmann, P. Periodic Structure Formation by Focused Electron-Beam-Induced Deposition. J. Vac. Sci. Technol., B: Microelectron. Process. Phenom. 2004, 22, 2504-2510.

(32) Bret, T.; Utke, I.; Hoffmann, P. Influence of the Beam Scan Direction during Focused Electron Beam Induced Deposition of 3DNanostructures. Microelectron. Eng. 2005, 78-79, 307-313.

(33) Bret, T.; Utke, I.; Hoffmann, P.; Abourida, M.; Doppelt, P. Electron Range Effects in Focused Electron Beam Induced Deposition of 3D-Nanostructures. Microelectron. Eng. 2006, 83, 1482-1486.

(34) Molhave, K.; Madsen, D. N.; Dohn, S.; Boggild, P. Constructing, Connecting and Soldering Nanostructures by Environmental Electron Beam Deposition. Nanotechnology 2004, 15, 1047-1053.

(35) Gazzadi, G. C.; Frabboni, S.; Menozzi, C. Suspended Nanostructures grown by Electron Beam-Induced Deposition of $\mathrm{Pt}$ and TEOS Precursors. Nanotechnology 2007, 18, 445709.

(36) Matsui, S. Focused-Ion-Beam Deposition for 3-D Nanostructure Fabrication. Nucl. Instrum. Methods Phys. Res., Sect. B 2007, 257, 758764.

(37) Winkler, R.; Szkudlarek, A.; Fowlkes, J. D.; Rack, P. D.; Utke, I.; Plank, H. Toward Ultraflat Surface Morphologies During Focused Electron Beam Induced Nanosynthesis: Disruption Origins and Compensation. ACS Appl. Mater. Interfaces 2015, 7, 3289-3297.

(38) Schmied, R.; Chernev, B.; Trimmel, G.; Plank, H. New Possibilities for Soft Matter Applications: Eliminating Technically Induced Thermal Stress during FIB Processing. RSC Adv. 2012, 2, 6932-6938.

(39) Toth, M.; Lobo, C.; Friedli, V.; Szkudlarek, A.; Utke, I. Continuum Models of fFocused Electron Beam Induced Processing. Beilstein J. Nanotechnol. 2015, 6, 1518-1540.

(40) Winkler, R.; Fowlkes, J.; Szkudlarek, A.; Utke, I.; Rack, P. D.; Plank, H. The Nanoscale Implications of a Molecular Gas Beam during Electron Beam Induced Deposition. ACS Appl. Mater. Interfaces 2014, 6, 2987-2995.

(41) Fernandez-Pacheco, A.; Serrano-Ramon, L.; Michalik, J. M.; Ibarra, M. R.; De Teresa, J. M.; O’Brien, L.; Petit, D.; Lee, J.; Cowburn, R. P. Domain Wall Conduit Behavior in Cobalt Nanowires Grown by Focused Electron Beam Induced Deposition. Sci. Rep. 2013, 3, 1492.

(42) Gazzadi, G. C.; Frabboni, S. Structural Transitions in Electron Beam Deposited Co-Carbonyl Suspended Nanowires at High Electrical Current Densities. Beilstein J. Nanotechnol. 2015, 6, 12981305.
(43) Keller, L.; Al Mamoori, M. K. I.; Pieper, J.; Gspan, C.; Stockem, I.; Schroder, C.; Barth, S.; Winkler, R.; Plank, H.; Pohlit, M.; Muller, J.; Huth, M. Direct-Write of Free-Form Building Blocks for Artificial Magnetic 3D-Lattices. Sci. Rep. 2018; In review.

(44) Esposito, M.; Tasco, V.; Cuscuna, M.; Todisco, F.; Benedetti, A.; Tarantini, I.; De Giorgi, M.; Sanvitto, D.; Passaseo, A. Nanoscale 3D-Chiral Plasmonic Helices with Circular Dichroism at Visible Frequencies. ACS Photonics 2015, 2, 105-114.

(45) Cordoba, R.; Sharma, N.; Kolling, S.; Koenraad, P. M.; Koopmans, B. High-Purity 3D-Nano-Objects Grown by FocusedElectron-Beam Induced Deposition. Nanotechnology 2016, 27, 355301.

(46) Kosters, D.; de Hoogh, A.; Zeijlemaker, H.; Acar, H.; Rotenberg, N.; Kuipers, L. Core-Shell Plasmonic Nanohelices. ACS Photonics 2017, 4, 1858-1863.

(47) Hoflich, K.; Yang, R. B.; Berger, A.; Leuchs, G.; Christiansen, S. The Direct Writing of Plasmonic Gold Nanostructures by ElectronBeam-Induced Deposition. Adv. Mater. 2011, 23, 2657-2661.

(48) Huth, M.; Porrati, F.; Dobrovolskiy, O. V. Focused Electron Beam Induced Deposition Meets Materials Science. Microelectron. Eng. 2018, 185-186, 9-28.

(49) Winkler, R.; Schmidt, F.-P.; Haselmann, U.; Fowlkes, J. D.; Lewis, B. B.; Kothleitner, G.; Rack, P. D.; Plank, H. Direct-Write 3DNanoprinting of Plasmonic Structures. ACS Appl. Mater. Interfaces 2017, 9, 8233-8240.

(50) Fowlkes, J. D.; Winkler, R.; Lewis, B. B.; Stanford, M. G.; Plank, H.; Rack, P. D. Simulation-Guided 3D-Nanomanufacturing via Focused Electron Beam Induced Deposition. ACS Nano 2016, 10, $6163-6172$

(51) Winkler, R.; Lewis, B. B.; Fowlkes, J. D.; Rack, P. D.; Plank, H. High-Fidelity 3D-Nanoprinting via Focused Electron Beams: Growth Fundamentals. ACS Appl. Nano Mater. 2018; Just accepted manuscript; complementary submission.10.1021/acsanm.8b00158

(52) Fowlkes, J. D.; Rack, P. D. Fundamental Electron-PrecursorSolid Interactions Derived from Time-Dependent Electron-BeamInduced Deposition Simulations and Experiments. ACS Nano 2010, 4, $1619-1629$.

(53) Sanz-Hernández, D.; Fernández-Pacheco, A. Modelling Focused Electron Beam Induced Deposition beyond Langmuir Adsorption. Beilstein J. Nanotechnol. 2017, 8, 2151-2161.

(54) Lewis, B. B.; Winkler, R.; Sang, X. H.; Pudasaini, P. R.; Stanford, M. G.; Plank, H.; Unocic, R. R.; Fowlkes, J. D.; Rack, P. D. 3DNanoprinting via Laser-Assisted Electron Beam Induced Deposition: Growth Kinetics, Enhanced Purity, and Electrical Resistivity. Beilstein J. Nanotechnol. 2017, 8, 801-812.

(55) Belianinov, A.; Burch, M. J.; Kim, S.; Tan, S.; Hlawacek, G.; Ovchinnikova, O. S. Noble Gas Ion Beams in Materials Science for Future Applications and Devices. MRS Bull. 2017, 42, 660-666.

(56) Lewis, B. B.; Mound, B. A.; Srijanto, B.; Fowlkes, J. D.; Pharr, G. M.; Rack, P. D. Growth and Nanomechanical Characterization of Nanoscale 3D-Architectures Grown via Focused Electron Beam Induced Deposition. Nanoscale 2017, 9, 16349-16356. 